\title{
NAFLD as a Sexual Dimorphic Disease: Role of Gender and Reproductive Status in the Development and Progression of Nonalcoholic Fatty Liver Disease and Inherent Cardiovascular Risk
}

\author{
Stefano Ballestri · Fabio Nascimbeni - Enrica Baldelli • \\ Alessandra Marrazzo · Dante Romagnoli · Amedeo Lonardo
}

Received: November 28, 2016 / Published online: May 19, 2017

(C) The Author(s) 2017. This article is an open access publication

\begin{abstract}
Nonalcoholic fatty liver disease (NAFLD) spans steatosis through nonalcoholic steatohepatis, cirrhosis, and hepatocellular carcinoma (HCC) associated with striking systemic features and excess cardiovascular and liver-related mortality. The pathogenesis of NAFLD is complex and multifactorial. Endocrine derangements are closely linked with dysmetabolic traits. For example, in animal and human studies, female sex is protected from dysmetabolism thanks to young individuals' ability to partition fatty acids towards ketone body production rather than very low density lipoprotein
\end{abstract}

Enhanced content To view enhanced content for this article go to http://www.medengine.com/Redeem/ 2128F060377C0C79.

Stefano Ballestri and Fabio Nascimbeni contributed equally and are joint first authors.

S. Ballestri

Azienda USL di Modena, Pavullo Hospital, Pavullo nel Frignano, Italy

F. Nascimbeni · D. Romagnoli · A. Lonardo Ospedale Civile di Baggiovara, Azienda

Ospedaliero-Universitaria di Modena, Modena, Italy

F. Nascimbeni $(\bowtie) \cdot$ E. Baldelli · A. Marrazzo Department of Biomedical, Metabolic and Neural Sciences, University of Modena and Reggio Emilia, Modena, Italy

e-mail: fabio.nascimbeni@unimore.it
(VLDL)-triacylglycerol, and to sex-specific browning of white adipose tissue. Ovarian senescence facilitates both the development of massive hepatic steatosis and the fibrotic progression of liver disease in an experimental overfed zebrafish model. Consistently, estrogen deficiency, by potentiating hepatic inflammatory changes, hastens the progression of disease in a dietary model of nonalcoholic steatohepatitis (NASH) developing in ovariectomized mice fed a high-fat diet. In humans, NAFLD more often affects men; and premenopausal women are equally protected from developing NAFLD as they are from cardiovascular disease. It would be expected that early menarche, definitely associated with estrogen activation, would produce protection against the risk of NAFLD. Nevertheless, it has been suggested that early menarche may confer an increased risk of NAFLD in adulthood, excess adiposity being the primary culprit of this association. Fertile age may be associated with more severe hepatocyte injury and inflammation, but also with a decreased risk of liver fibrosis compared to men and postmenopausal status. Later in life, ovarian senescence is strongly associated with severe steatosis and fibrosing NASH, which may occur in postmenopausal women. Estrogen deficiency is deemed to be responsible for these findings via the development of postmenopausal metabolic syndrome. Estrogen supplementation may at least theoretically protect from NAFLD development and progression, as suggested by 
some studies exploring the effect of hormonal replacement therapy on postmenopausal women, but the variable impact of different sex hormones in NAFLD (i.e., the pro-inflammatory effect of progesterone) should be carefully considered.

Keywords: Fibrosis; Hormones; Inflammation; Man; Menarche; Menopausal status; NASH; Physiopathology; Sex; Steatosis; Women

\section{INTRODUCTION}

Nonalcoholic fatty liver disease (NAFLD) encompasses the whole spectrum of (predominantly) steatogenic liver disorders spanning steatosis through nonalcoholic steatohepatitis (NASH), cirrhosis, and hepatocellular carcinoma (HCC) [1-3], associated with striking systemic features $[4,5]$ and excess cardiovascular and liver-related mortality [6-9]. Histologically indistinguishable from alcoholic liver disease, and nevertheless observed in the nonalcoholic individual [10], NAFLD is closely linked with insulin resistance (IR) [11] and, bidirectionally, with the metabolic syndrome (MetS) of which it may be both a cause and a consequence $[12,13]$. A leading cause of chronic liver disease worldwide and affecting one out of four individuals in the European and north-American general populations [14], NAFLD is highly prevalent in certain groups of individuals carrying either the full-blown or individual traits of the MetS [15]. Moreover, NAFLD carries an excess of health-related expenditures owing to its close connections with progressive liver disease and cardio-metabolic morbi-mortality [9, 16-19].

The pathogenesis of NAFLD has variably been conceptualized as two-hit [20], a one-hit [11], or a multistep process [21], and the last is presently the most widely accepted pathogenic model. In its original definition, a first hit leads to steatogenesis and a second one to fibrosis [22]. However, metabolic factors, and particularly IR, invariably account for most of the elementary NAFLD histologic lesions in humans, indirectly supporting the outdated and yet conceptually more parsimonious "one-hit" theory $[11,12]$.

Evidence both in animals and humans supports the notion that female sex is protected from dysmetabolic traits thanks to young individuals' ability to partition fatty acids towards ketone body production rather than very low density lipoprotein (VLDL)-triacylglycerol [23], and to sex-specific browning of white adipose tissue which contributes in protecting female mice from experimental NAFLD associated with methionine choline deficient diet [24].

In 1980, Ludwig reported that NASH was common among elderly women with metabolic comorbidity [25]. However, we now know that NAFLD more often affects men $[15,26]$ and that premenopausal women are equally protected from NAFLD development as they are from cardiovascular disease (CVD) [27, 28]. Recent studies in the overnourished zebrafish model have shown that ovarian senescence, via hypoestrogenemia, facilitates both the development of massive hepatic steatosis and the fibrotic progression of liver disease [29]. Consistently, estrogen deficiency, by potentiating hepatic inflammatory changes, hastens the progression of disease in a dietary model of NASH developing in ovariectomized mice fed a cholesterol-rich hyperlipidic diet [30]. Collectively, these findings suggest that hormonal changes, rather than those multiple physiologic derangements associated with aging per se [31], are a major determinant of progressive NAFLD in human menopause.

Obesity and obesity-related diseases, such as type 2 diabetes (T2D), MetS, and atherosclerosis, are complex conditions driven by genetic and environmental factors, in which a sexual dimorphism has been clearly established. Here, we have reviewed current evidence suggesting that NAFLD is a sexually dimorphic condition, too. We hypothesized that the higher incidence of disease in men and the worse outcome in postmenopausal women, i.e., the "sexual dimorphism" of NAFLD, might offer clues useful in expanding our understanding of the pathogenesis and providing hints for prevention and treatment of NAFLD. Given the potential research and clinical implications of sexual dimorphism in NAFLD, we carried out a 
systematic review of the literature by using the following keywords: steatosis OR fatty liver AND gender OR sex OR menopause. On these grounds, this narrative review is aimed to highlight how sex modulates the development and progression of NAFLD and to pinpoint what the role of menarche and menopause is. Special emphasis is conferred to CVD risk. The relationship of polycystic ovary syndrome (PCOS) to NAFLD has recently been covered elsewhere [32] and is outside the scope of this review.

\section{Compliance with Ethics Guidelines}

This article is based on previously conducted studies and does not involve any new studies of human or animal subjects performed by any of the authors.

\section{EPIDEMIOLOGY OF NAFLD}

Gender and reproductive status modulate the risk of developing NAFLD and NASH with/ without advanced fibrosis [15].

\section{Risk of NAFLD}

\section{Incidence}

Evidence from longitudinal studies suggests that the incidence of NAFLD is higher in the male as compared to the female gender (Table 1) [33-43]. One study investigating the incidence of NAFLD in women as a function of reproductive status found incidence to be higher in menopausal (7.5\%)/postmenopausal (6.1\%) women as compared to premenopausal (3.5\%) women. However, postmenopausal status was associated with an increased risk of incident NAFLD at univariate but not at multivariate analysis adjusted for age, metabolic syndrome, and weight gain [37]. The incidence of NAFLD in women taking hormonal replacement therapy (HRT) $(5.3 \%)$ was higher than in premenopausal (3.5\%) women but lower than in menopausal women (7.5\%). HRT was not associated with increased risk of incident NAFLD either at univariate or at multivariate analysis [37]. Indeed other longitudinal studies suggest that estrogens are a protective factor for the development of NAFLD. An Italian multicentric study on 5408 healthy women who had had hysterectomies, randomly assigned to receive tamoxifen (an estrogen inhibitor) or placebo for 5 years, showed that tamoxifen was associated with higher risk of development of NAFLD/NASH especially in overweight/obese women [44]. Finally a small double-blind, randomized placebo-controlled trial on 50 women with T2D showed that HRT containing low-dose estradiol and norethisterone significantly reduced serum levels of liver function enzymes, potentially owing to a reduced liver fat accumulation [45].

Overall, data from epidemiological longitudinal studies have shown a key role of weight gain, presence of MetS and its single traits as independent predictors of the development of NAFLD [33-43]. Notably, some studies have found that male sex was associated with incident NAFLD independently of age and metabolic factors [33, 35, 41]. Moreover, two Asian studies have reported a specific role of age according to gender and reproductive status in modulating the risk of incident NAFLD. One study showed that age was an independent predictor for developing NAFLD only in females [34] and the other one reported that age increased the risk for incident NAFLD in premenopausal but not in postmenopausal women [37].

\section{Prevalence}

Despite preliminary studies reporting a higher risk of NAFLD in females, a large body of evidence now definitely supports the notion that the prevalence of NAFLD is higher in men than in women and that gender-specific differences exist in relation to age (Table 1) $[33,37,41,46-77]$. Conflicting with the above notion, two large sample community Iranian studies reported a higher prevalence of NAFLD in women than in men $[70,74]$. However, these findings might, at least in part, be accounted for by women having markedly higher rates of prevalence of obesity compared to men in these studies. Consistent with the view reported above, a higher prevalence of fatty liver has 


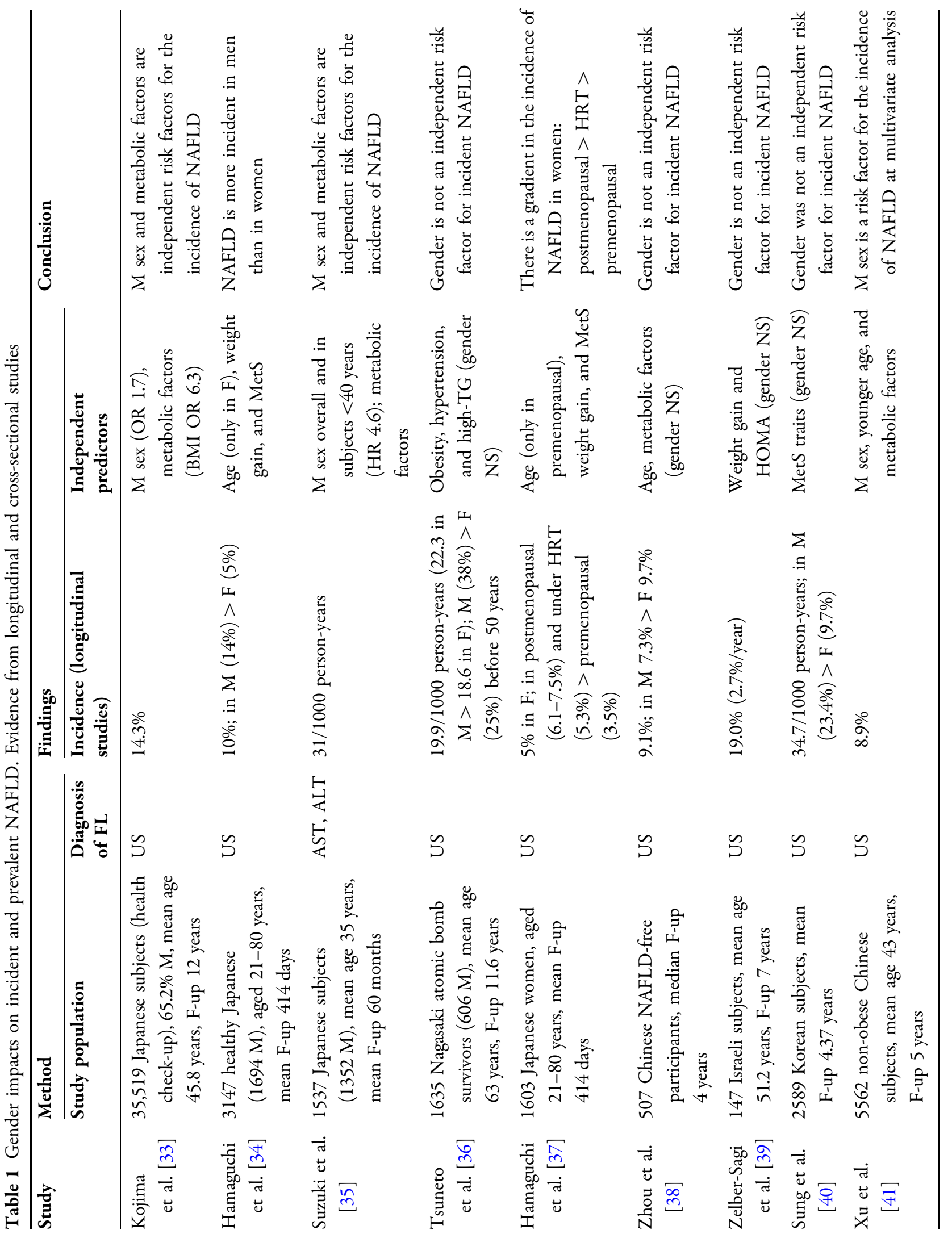




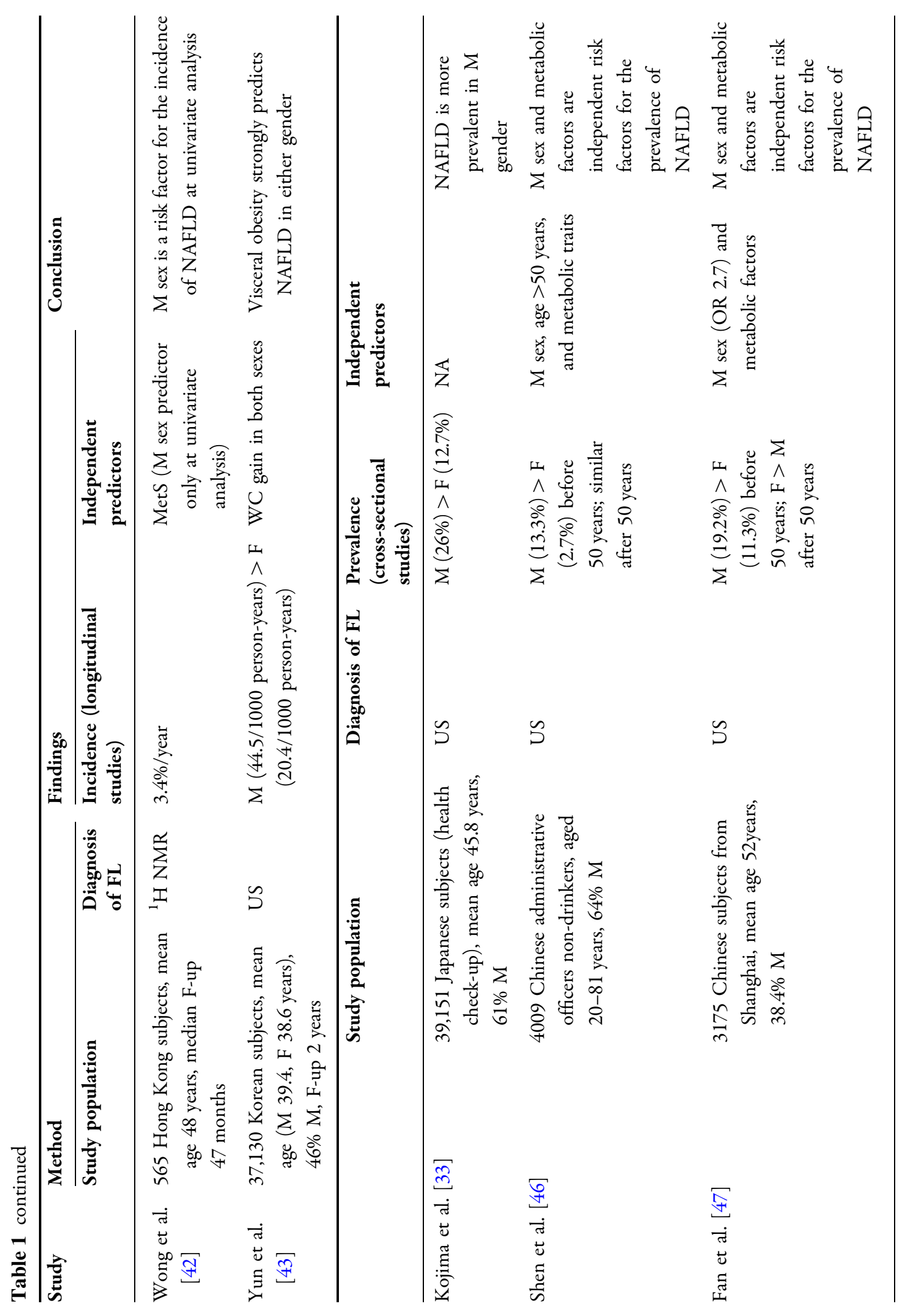




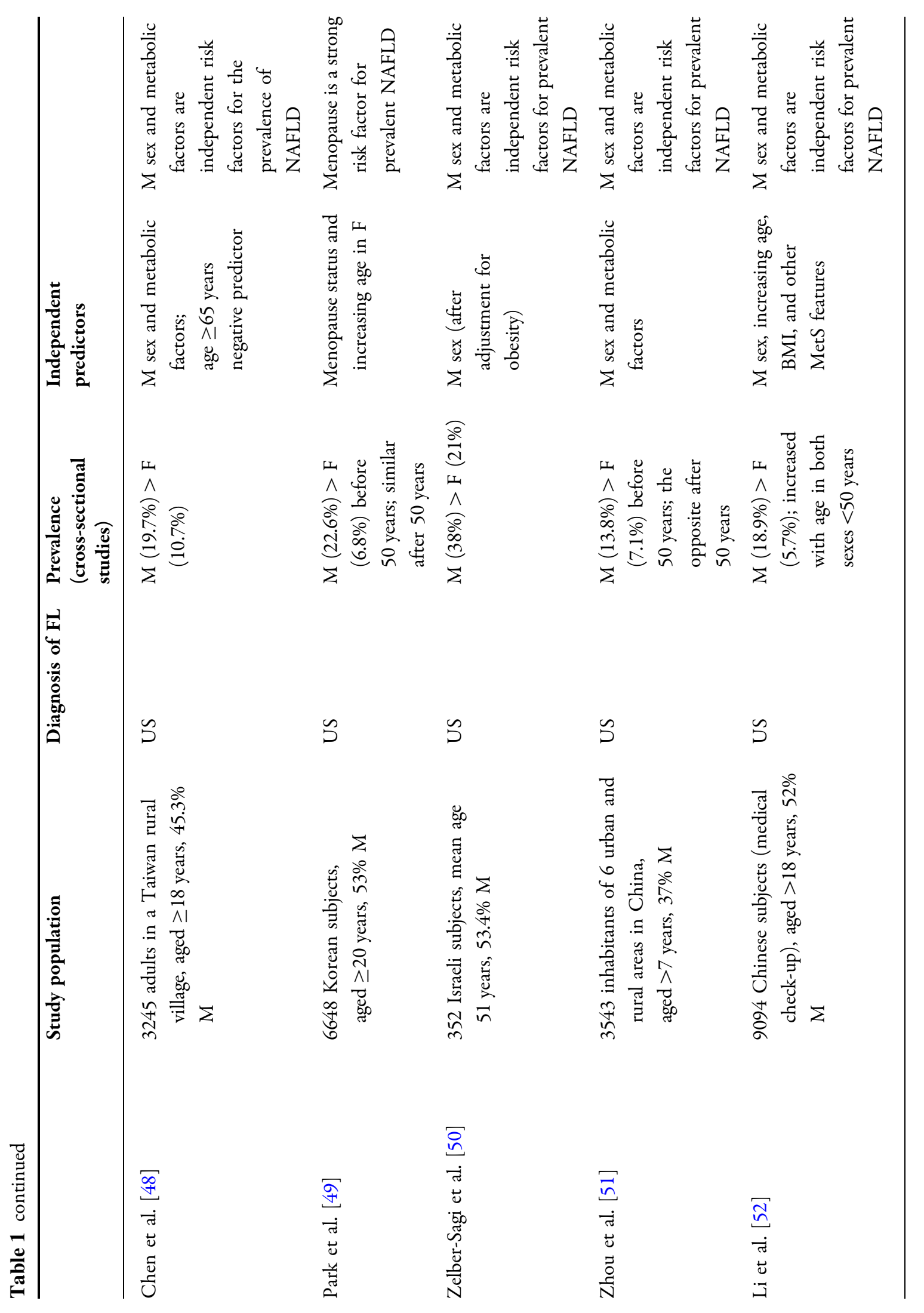




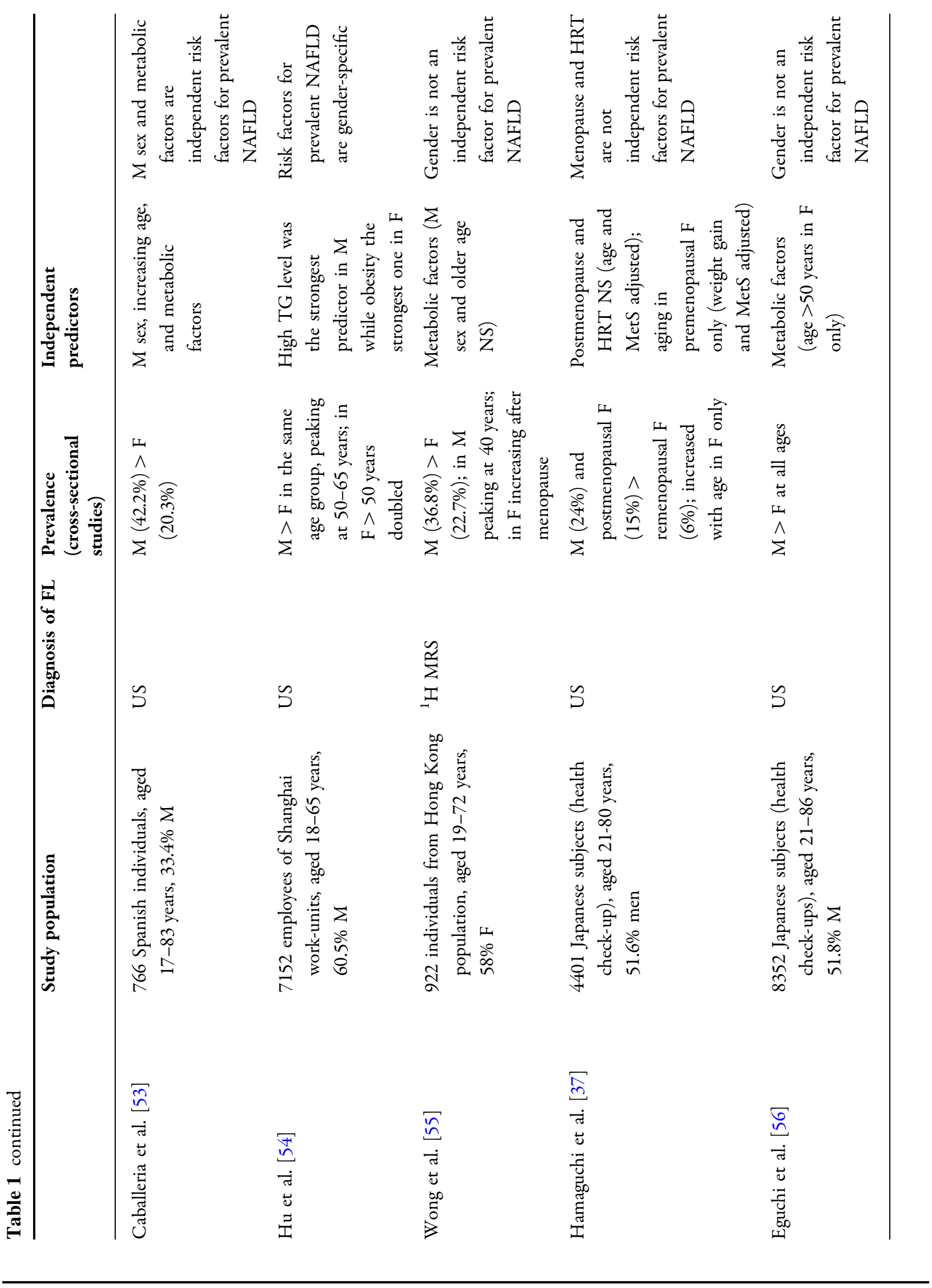




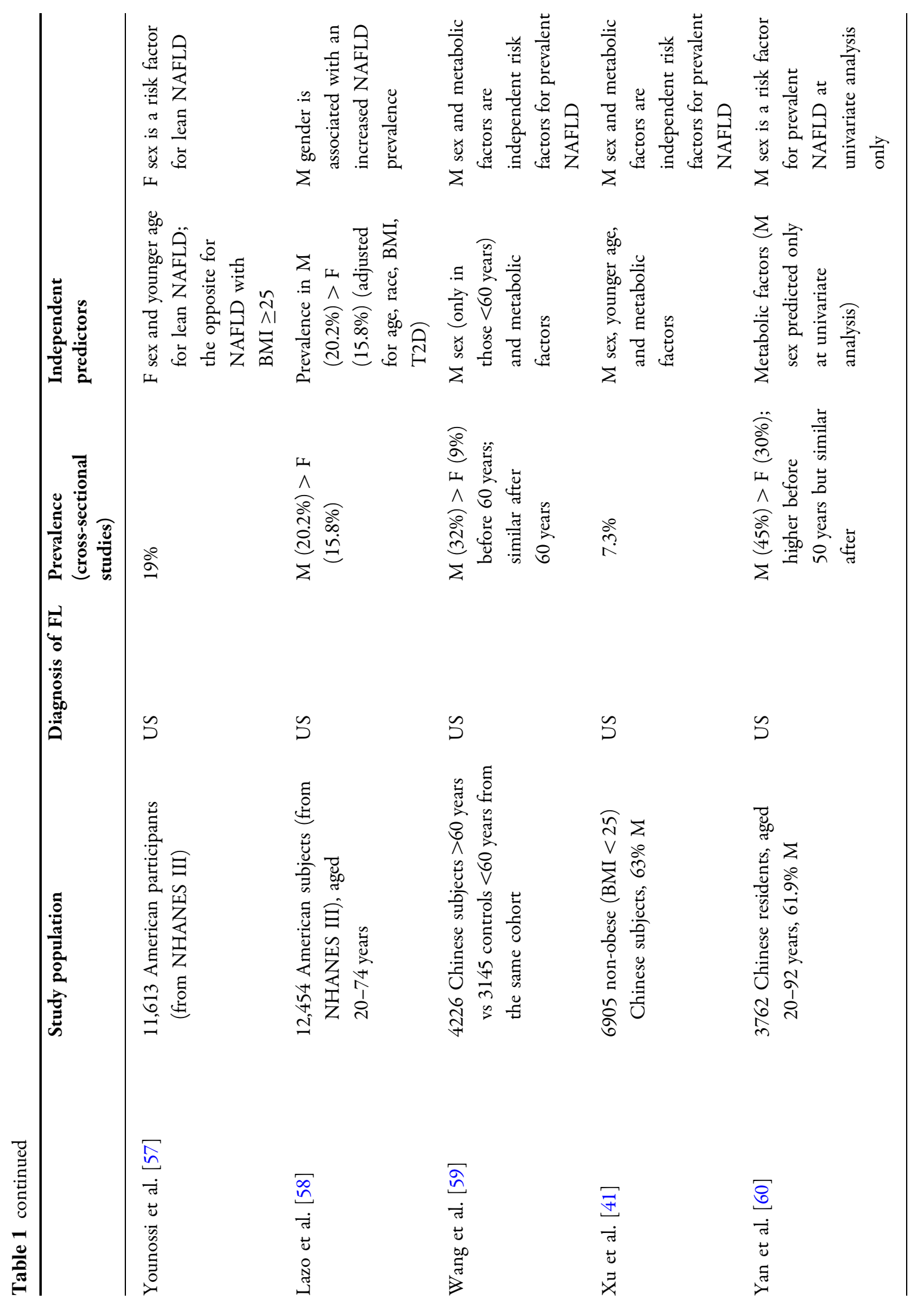




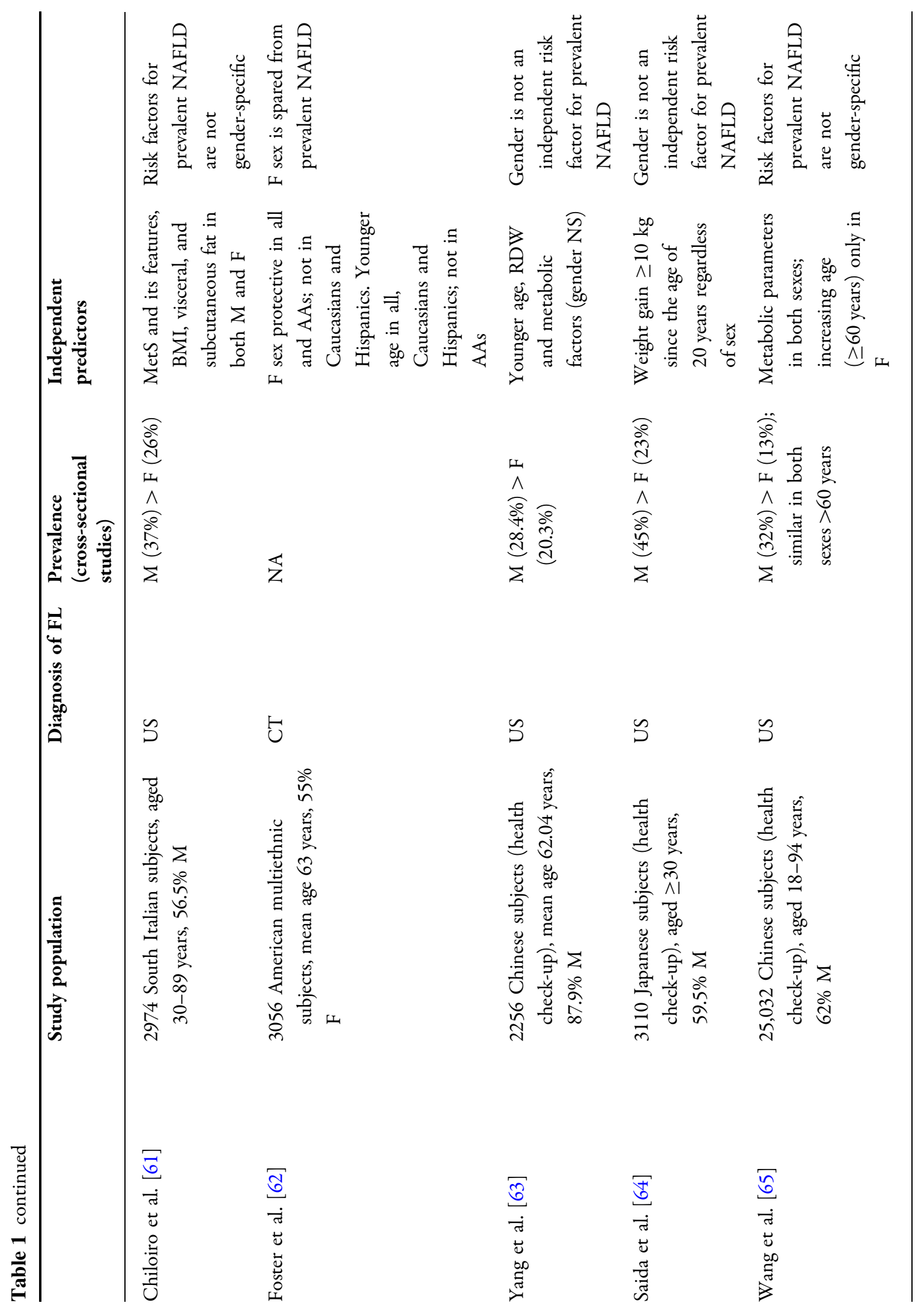




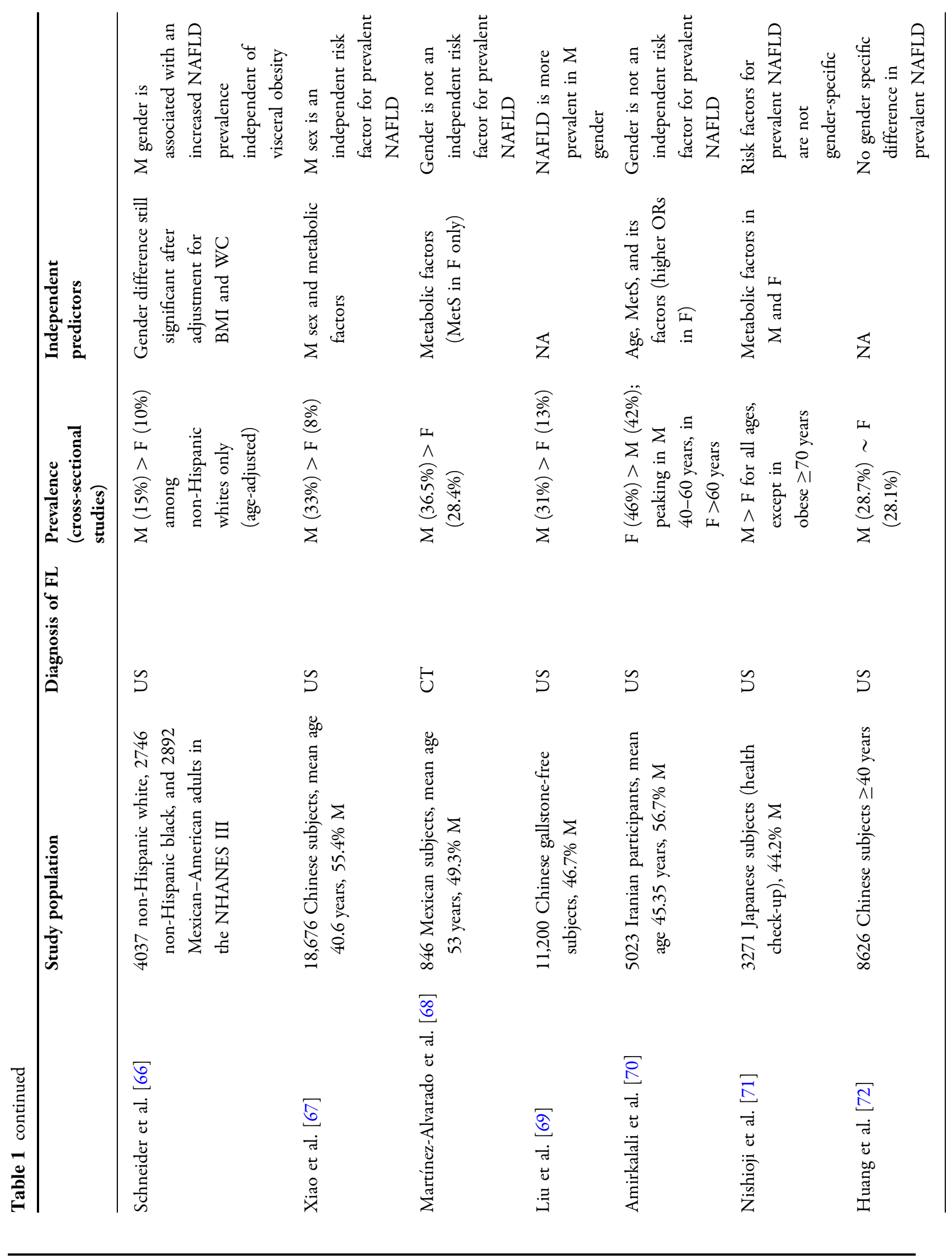




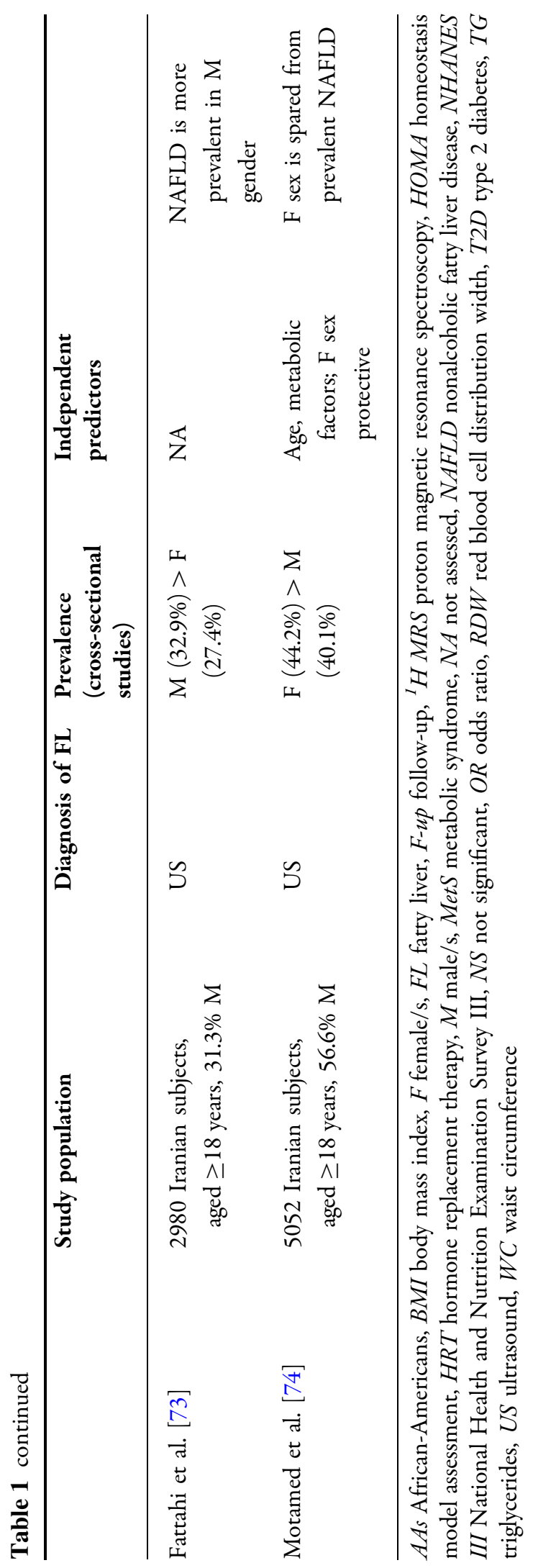

been also observed in male obese children and adolescents than in female ones [78].

Men commonly display an increasing prevalence of NAFLD during adulthood from young to middle-age, describing an "inverted U-shaped curve" which starts declining after the age of 50-60 years $[47,56]$. In women of fertile age, the prevalence of NAFLD is lower than in men owing to the putative protective effect of estrogens which, however, wanes after the menopause. Accordingly, the prevalence of NAFLD in women rises after the age of 50 years, peaks at $60-69$ years, and declines after 70 years $[47,54,56]$. As a result of this, after the fifth decade, postmenopausal women compared to men of the same age have a similar $[46,49,60,65]$ or even higher $[47,51]$ prevalence of NAFLD. In agreement with these findings, a multicenter study from northern Italy found that men with NAFLD were approximately 10 years younger than women with this condition [79], a finding compatible with the hypothesis that premenopausal women are "protected" from developing NAFLD. Conversely, NAFLD is more prevalent in postmenopausal [37, 80-82] and PCOS-affected women than premenopausal ones [80]. Consistently, a large cross-sectional population-based survey in northeast Germany on 808 women aged 40-59 years showed that menopause status was independently associated with hepatic steatosis after adjustment for metabolic factors [83]. Interestingly, women with NAFLD exhibit a significantly lower concentration of serum estradiol, which is the principal active estrogen, than NAFLD-free (premenopausal, postmenopausal, and PCOS) controls [80]. A lower prevalence of NAFLD, as well as of MetS, has been reported in postmenopausal women receiving HRT compared to those not receiving it, which suggests that HRT probably protects from NAFLD. Moreover, in this study postmenopausal women with NAFLD who received HRT had lower frequency of insulin resistance as well as MetS and showed reduced serum levels of liver enzymes and ferritin [84].

Most studies using multivariate analysis have shown that male sex is associated with an increased prevalence of NAFLD independently of age and metabolic conditions 
$[41,46,48,50,51,53,58,62,63,66,67,74]$. Some studies have shown an independent, positive association between NAFLD and increasing age in both sexes [46, 50-53, 70, 74] or in females only [65], while others reported an inverse association [41, 48, 62, 63]. At variance, other studies have shown that sex differences in the prevalence of NAFLD were mostly accounted for by metabolic factors $[36,37,55,56,60$, $63,64]$. Finally, some studies failed to investigate the role of gender given that a separate multivariate analysis according to sex was not performed [34, 49, 54, 56, 61, 65, 68, 70, 71].

In addition to and independent of the role of MetS and its components, menopausal status $[49,83,85]$ and increasing age $[49,56,82]$ have all been consistently identified as strong risk factors for NAFLD in women. Only in a few studies was the association between menopausal status and NAFLD no longer significant after adjustment for age and metabolic factors $[37,81]$, indicating that menopause predisposes to developing NAFLD via incident dysmetabolic traits which typically appear in the postmenopausal age.

A recent cross-sectional study conducted in a population of postmenopausal women concluded that MetS, abdominal obesity, and IR are risk factors for the development of NAFLD while higher adiponectin levels protect from developing it [86]. These findings indicate that NAFLD modifiers in this specific population of women closely mirror those found in the general population.

In lean subjects [body mass index (BMI) less than $25 \mathrm{~kg} / \mathrm{m}^{2}$ )] NAFLD has been associated with younger age $[41,57]$ and either male [41] or female gender [57].

\section{Risk of NASH and Fibrosis Progression}

One large sample study based on an electronic health file database reported that the risk of "recorded" NAFLD/NASH diagnosis increased linearly with BMI, was higher in males than females and in those with T2D [87]. However, the proportion of true NASH cases among the "recorded" diagnosis of NAFLD/NASH was unclear.
A longitudinal study in subjects with biopsy-proven NAFLD found that sex was not an independent risk factor for the progression of fibrosis [88]. In agreement, a systematic review has shown that age and hepatic necroinflammation are the only independent predictors of the development of advanced fibrosis in NASH patients, while other parameters such as MetS features and sex are not [89].

However, conflicting with the above studies, data from cross-sectional studies (Table 2) $[29,57,65,90-97]$, which are based, in the majority of cases, on a histological diagnosis of $\mathrm{NASH}$, tend to suggest that the risk of NASH and advanced fibrosis is indeed higher in females than males independent of metabolic factors $[65,90,93,94]$, and only a few studies conflict with the above findings [91, 92, 95].

As is easily foreseeable, obese and postmenopausal women, compared to premenopausal and non-obese women, suffer from a remarkably higher prevalence of NAFLD and NASH as a result of a worse metabolic profile [82]. At variance, a recent study reported that, compared to men and postmenopausal women, the risk of lobular inflammation and hepatocyte injury was significantly increased both in premenopausal women and in those taking synthetic hormones such as oral contraceptives and HRT [97]. Given the supposed effects of estrogens on metabolic health and liver injury, the findings of this study appear counterintuitive. It is noteworthy that the study by Yang et al. has several limitations, including some important sources of potential bias, such as the restricted enrollment at tertiary academic centers, menopausal category and synthetic hormone use were self-reported, and information on cumulative estrogen and/or progesterone exposure and serum hormonal levels were lacking. Of note, the authors highlight that, despite increased liver injury and inflammation, premenopausal women were at decreased risk of liver fibrosis compared with men and postmenopausal women. Moreover, sensitivity analyses separately assessing the impact of progesterone use and estrogen use clearly suggested that only the former was associated with liver damage. Collectively these findings provide 


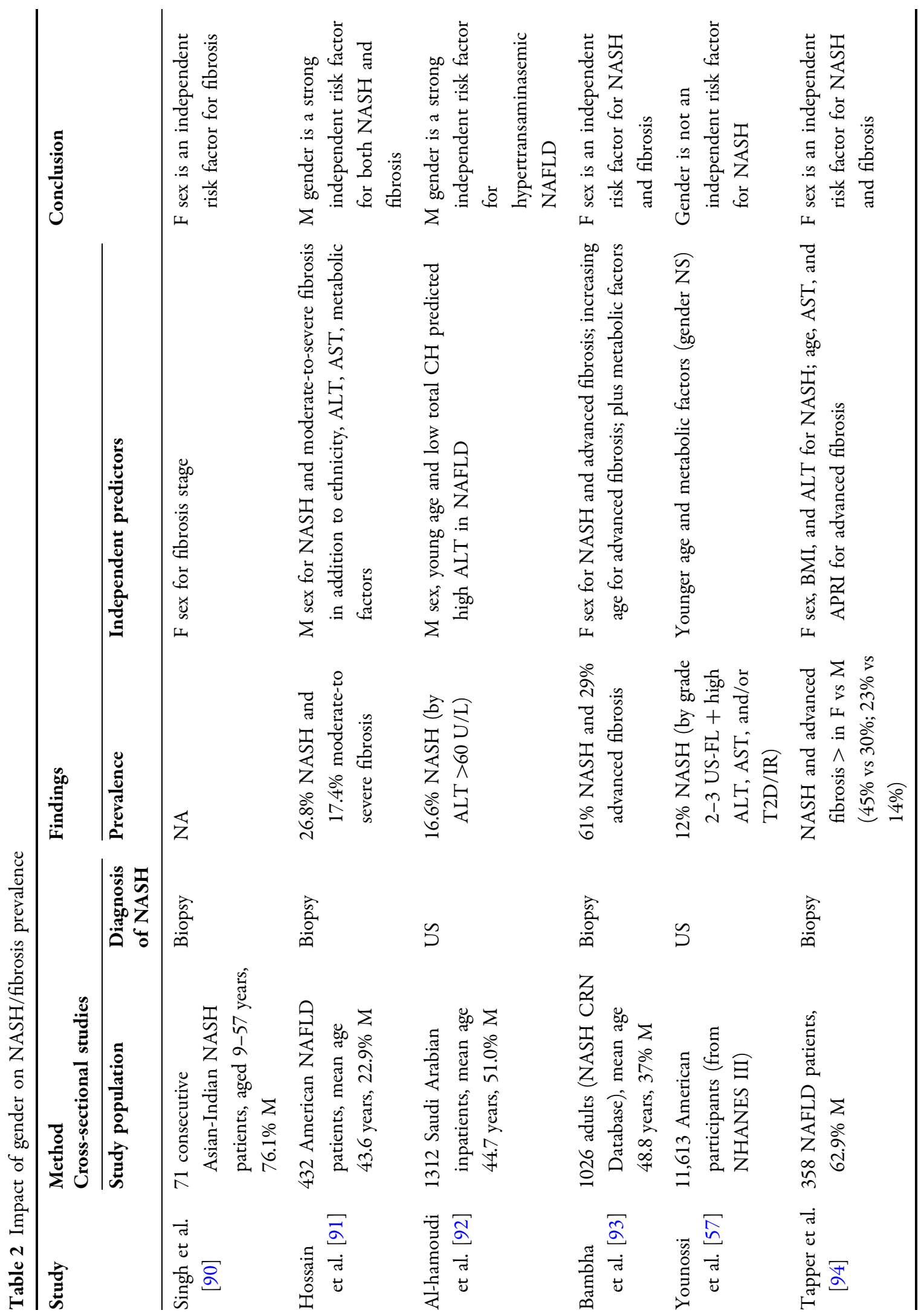




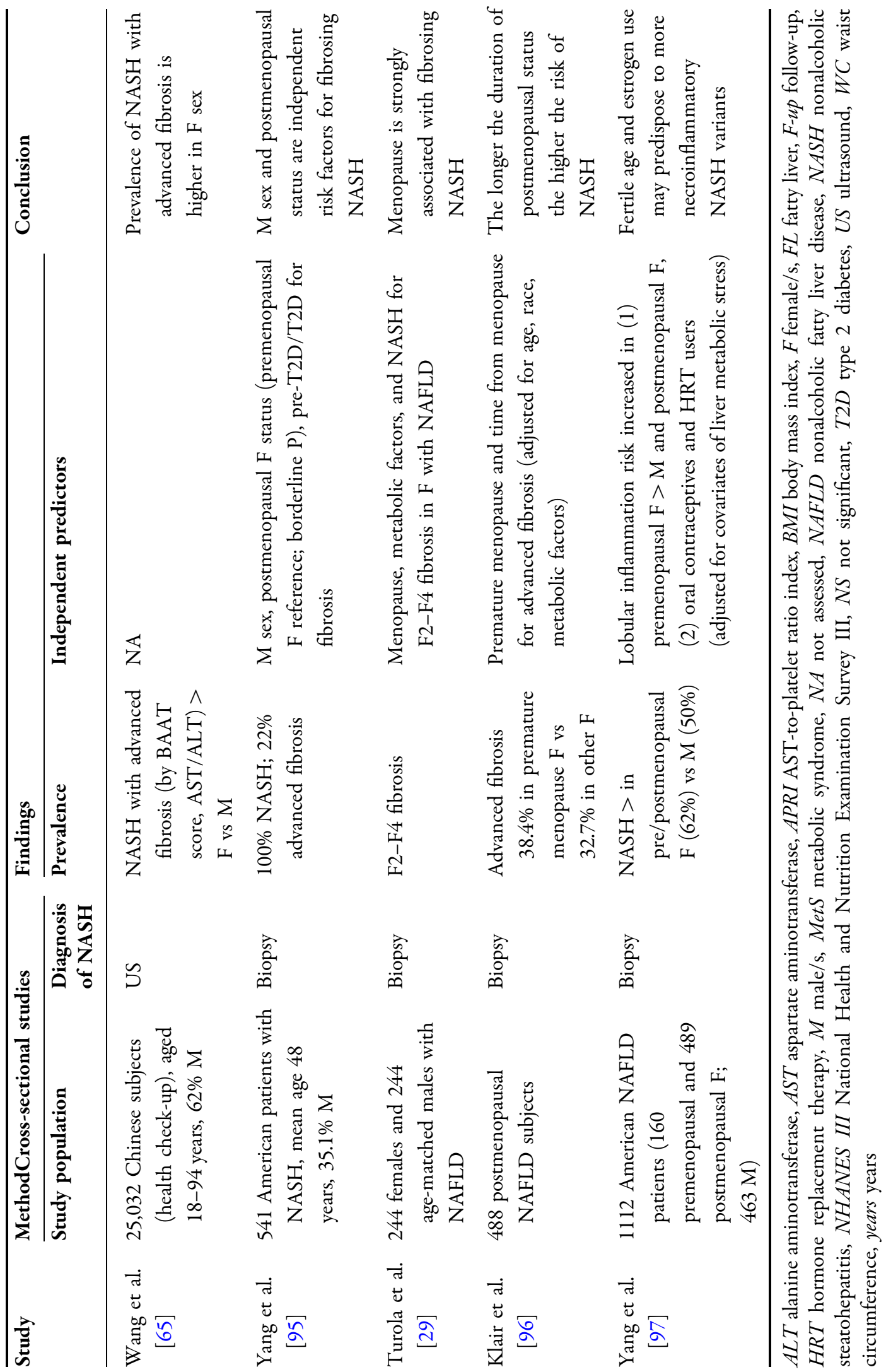


novel hints regarding a potential multifaceted impact of sex hormones in NAFLD.

Consistent with the hypothesis that estrogens do exert a beneficial effect on NAFLD, menopause has been independently associated with significant fibrosis both in women with NAFLD and in an experimental zebrafish steatosis model [29]. A recent study has shown that men display a higher risk of advanced fibrosis compared to premenopausal women, while after menopause both sexes show a similar severity of liver fibrosis, suggesting that estrogens protect from the development of fibrosis [95]. A subsequent study limited to non-obese women with biopsy-proven NAFLD confirmed that, even in non-obese NAFLD patients, postmenopausal women still had more severe fibrosis, when compared to premenopausal subjects [98]. Accordingly, a study conducted in 488 postmenopausal women with biopsy-proven NAFLD has shown that, independently of age and metabolic factors, the longer the estrogen deficiency in postmenopausal status is (i.e., premature menopause and time from menopause), the higher the risk of fibrosis is [96].

The role of gender in influencing liver-related mortality in NAFLD is still uncertain, although some studies have reported that men are at an increased risk [99-101].

\section{Risk of Hepatocellular Carcinoma}

A universal feature of HCC is the striking male prevalence, with a male/female ratio averaging $2: 1$ to $7: 1$; the latter proportion is more often found in HBV-related HCC [102]. Nevertheless, compared to viral etiologies of HCC, NAFLD-related HCC was found to be associated with the lowest male/female ratios in one study [103]. NAFLD-related HCC may also occur in non-cirrhotic livers, but this seems to be more likely in men [104]. The sexual dimorphism in HCC is also maintained regarding prognosis, with women showing better survival rates [102, 105]. However, menopause seems to attenuate these advantages [102, 105].

In summary, the incidence of NAFLD is higher in men than in women, and some longitudinal studies indicate that male gender is an independent predictor of NAFLD development. The prevalence of NAFLD is globally higher in men than women, but after menopause women display a similar or even a higher prevalence compared to men, a finding supporting a protective effect of estrogens. In cross-sectional studies, male gender and menopausal status have often been associated with the risk of NAFLD, independent of age and metabolic factors. The prevalence of NASH and advanced fibrosis has been found to be higher in postmenopausal women than in men; however, longitudinal studies have failed to support a role for gender in influencing the progression of liver fibrosis. Finally, HCC is definitely more common in men than in women in cases due to viral etiology; NAFLD may probably lower the male/female ratio in the risk of developing HCC and it is possible that gender affects the prognosis of HCC.

\section{NAFLD AND CVD}

NAFLD is increasingly recognized as a multisystem disease [5]. A growing body of evidence suggests that NAFLD (assessed by liver enzymes, imaging, or biopsy) is associated with increased incidence and prevalence of subclinical and clinical CVD, mainly coronary heart disease (CHD), independently of age, gender, and metabolic factors, as recently reviewed in detail elsewhere $[106,107]$. In the general population, male sex is more prone to incident CHD under the age of 50 years compared to women but, after menopause, the incidence in women dramatically increases to approach that of men [108].

\section{Gender-Specific Risk of CVD in Studies on NAFLD and CVD}

Several longitudinal studies investigating the association between CVD and NAFLD have reported multivariate analysis models, which have been adjusted for multiple confounders, including sex; as a result of this, the influence of gender in the risk of CVD cannot be evaluated in such studies (reviewed in [106, 107]). A large 
population-based American study (NHANES III) found that NAFLD assessed with ultrasound, together with male sex, age, race, and metabolic factors independently predicted incident CVD [109]. Another study addressing CHD as a prespecified outcome found that patients with NAFLD had a higher 10-year risk for CHD, as calculated by the Framingham risk score, than the matched control population. This estimated risk was higher in men than in women and more CVD events were reported among men than women at the end of 10 year follow-up, although this finding was not statistically significant [110].

Many cross-sectional studies in which the diagnosis of NAFLD was based on liver imaging studies (either ultrasound or CT scanning) showed an independent association of male sex with coronary artery calcifications [111] as well as significant CHD [112-115].

\section{Gender Differences in the Association Between NAFLD and CVD}

Studies in which the diagnosis of NAFLD was based on surrogate indices, such as otherwise unexplained raised liver enzymes, found that sex modulates the association of NAFLD with incident CVD/mortality. For example, most population-based cohort and meta-analytic studies reported an independent association between raised GGT and incident CVD in both sexes [116]; conversely, a population-based cohort study from Germany found that increased GGT was associated with higher risk of all-cause and CVD mortality only in men but not in women, and this association was stronger in men who also had ultrasound scanning findings compatible with steatosis [101]. Increased ALT has been variably associated with incident CVD either in both sexes $[117,118]$ or in men only [119]. A recent study found that ALT levels independently predicted insulin sensitivity only in women, suggesting that this gender-specific association might explain the sex difference in the predictive role of increased ALT for CVD [120]. Finally, a recent large Korean cross-sectional study reported that men had more prevalent ultrasonographic fatty liver disease, carotid plaques, and increased carotid intima-media thickness (IMT) values than women, but ultrasonographic fatty liver disease independently predicted subclinical carotid atherosclerosis (IMT and plaques) in women only [121].

No gender difference has been reported in the association between NAFLD assessed with liver ultrasound and incident CVD/mortality. A study based on a national Danish registry showed that patients with a hospital discharge diagnosis of NAFLD had a higher all-cause mortality, including liver and CVD related, which was similar among sexes [122]. A study conducted in a community-based Japanese cohort of 1637 apparently healthy subjects found that the diagnosis of NAFLD based on ultrasound findings was a predictor of CVD in both men and women [123].

The only study carried out in postmenopausal women found a significant correlation between NAFLD (based on CT scanning findings) and prevalence of coronary artery calcification (CAC); however, NAFLD was not independently associated with CAC in these postmenopausal women [124].

In summary, male patients carry an increased risk of CVD; moreover, NAFLD seems to be associated with CVD independently of metabolic factors in both sexes. Few data are available in postmenopausal women and studies should specifically be conducted to ascertain whether NAFLD is a specific/independent cardiovascular risk factor in this population of patients.

\section{ROLE OF GENDER, REPRODUCTIVE STATUS, AND AGE IN THE HETEROGENEITY OF NAFLD PATHOBIOLOGY}

Although NAFLD may be found in either gender from infancy to old adulthood, gender and reproductive status modulate the susceptibility to development and progression of disease [125]. Indeed age, sex, and fertility exert a variable impact on those general pathogenic mechanisms which are involved in NAFLD. 
Here we will specifically examine how body fat distribution, obesity and local hypercorticolism, steatogenesis and lipidomics, oxidative stress and antioxidant mechanisms, endotoxins, immune response, and fibrogenesis are affected by gender and sex hormones as a result of reproductive status and age.

\section{Factors Associated with NAFLD are Different in Men and in Women}

A pioneering study supporting the notion that gender-dimorphic risk factors are associated with NAFLD was published in 2000. This study, by evaluating 199 individuals, found that elevated BMI was an independent predictor of fatty liver in either sex. Glucose area under the curve and a central-type body fat distribution predicted fatty liver only in women [126]. Similarly, insulin sensitivity has also been reported to be strongly associated with gender-dimorphic risk factors, i.e., fasting insulin and leptin levels (but none of the liver enzymes) in men versus BMI and ALT in women [120]. Of note, Suzuki et al. demonstrated that the associations between anthropometric measures (regional adiposity) and degree of fibrosis clearly differ between premenopausal women and postmenopausal women/men [127].

\section{NAFLD Epidemiology} and Physiopathology are Modulated by Age at Menarche and Postmenopausal Status

In women, a complex interaction including genetic polymophisms, dietary habits, endogenous sex hormones, age at menarche, menopausal status, dysmetabolic traits, and HRT modulates the risk of developing NAFLD, NASH, and fibrosis [84, 128-130].

Early menarche has been associated with higher alanine aminotransferase, C-reactive protein, triglyceride levels, BMI, waist circumference, adult diabetes, cardiovascular morbidity and mortality, advanced liver disease, and HCC [131]. A recent Chinese study conducted in postmenopausal women has identified early menarche as a potential risk factor for NAFLD later in life; consistently, late menarche protects from NAFLD [130]. These associations were significantly attenuated after adjustment for current BMI or HOMA-IR, suggesting that obesity and insulin resistance may partly mediate the association between age at menarche and NAFLD [130]. The biological mechanism linking early menarche with increased risk of NAFLD is far from being clearly elucidated. It has been suggested that early maturation may determine a longer duration of positive energy balance and a greater accumulation of body fat [132]. Consistently, a large cross-sectional study among middle-aged Korean women confirmed that the inverse association between age at menarche and NAFLD was partially mediated by adiposity [133]. Again, a recent study from the American CARDIA cohort showed that early menarche was associated with NAFLD and visceral and subcutaneous abdominal ectopic fat depots in middle adulthood; these associations were attenuated after adjustment for weight gain between young and middle adulthood [131]. Finally, a Chinese study suggested that the presence of central obesity and MetS, but not NAFLD, after menopause was predicted by longer duration of menstruation and early menarche [134].

Compared to the fertile age, menopause increases the risk of NAFLD and liver fibrosis [135] via long-standing estrogen deficiency associated with ovarian senescence and dysmetabolic features such as T2D, hypertriglyceridemia, and central obesity [29, 81]. Consistently, HRT protects from NAFLD development [84], and oophorectomy in young women with endometrial cancer independently increases the risk of NAFLD together with the development of T2D and hypercholesterolemia [136]. These findings are in agreement with a study suggesting that, in $\mathrm{HCV}$ infection, increasing severity of fibrosis is associated with a higher BMI, advanced steatosis, and the menopause and that, conversely, menopausal women receiving HRT exhibit a lower stage of fibrosis [137]. Collectively, these data support the notion that estrogens have antifibrogenic properties in humans. This antifibrotic activity may occur by triggering anti-inflammatory, antioxidant, and antiapoptotic molecular 
pathways [138], which are mimicked by exercise training [139].

Of concern, however, young women in their reproductive age and those exposed to female synthetic hormones (oral contraceptive or HRT) are not completely spared the risk of developing NAFLD and, indeed, they tend to have more severe hepatocyte injury and inflammation. Notably, despite the possibility of enhanced hepatocellular damage, premenopausal women have been consistently reported at decreased risk of liver fibrosis compared to men and postmenopausal women. Moreover, sex hormones exert complex and variable effects on human NAFLD; indeed, the detrimental pro-inflammatory impact may be conveyed by progesterone, but not estrogen [97].

Table 3 summarizes the physiological role of estrogen, progesterone, and androgens according to gender, age, reproductive status, and obesity [140-157].

\section{Obesity and Local Hypercortisolism}

Obesity, which is closely linked with NAFLD, mimics hypercortisolism. It is of interest that despite the prevalence of obesity being higher among women than men, the former are somewhat protected from the associated cardiometabolic consequences; however, this wanes after menopause, suggesting a role for estrogens. Mouse models suggest that sexually dimorphic expression and activity of glucocorticoid metabolizing enzymes may have a role in the differential metabolic responses to obesity in males and females [158].

Biochemical, genetic, and therapeutic stud-

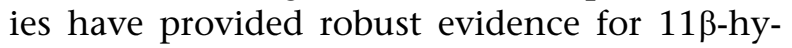
droxysteroid dehydrogenase type 1 (11 $\beta$-HSD1) being key in the pathogenesis of NAFLD [159-161]. It is of interest, therefore, that $11 \beta$-HSD1 gene expression is regulated in a tissue-specific and sexually dimorphic manner. In particular, intact rats exhibit hepatic $11 \beta-H S D 1$ mRNA levels 18 -fold lower in the female than the male [162].

\section{Body Fat Distribution}

Regional adiposity displays a typical sexual dimorphism in humans. Women have a larger capacity to store fat in the subcutaneous compartment [163]. Men generally have twice as much visceral fat compared to women for any given fat mass value [164]. This is relevant given that, compared to subcutaneous adipose tissue depots, visceral adipose tissue depots in general display greater secretory capacity and a more pro-inflammatory profile [165]. Moreover, visceral adipose depots release free fatty acids (FFAs) directly into the portal blood and thus potentially overload the liver [166]. Not surprisingly, those phenotypes which occur whenever, due to hormonal effect, peripheral adiposity is relatively restricted and visceral adiposity is expanded (i.e., male obesity and postmenopausal women) have all been associated with NASH, and fibrosing NASH [127, 167-169].

\section{Steatogenesis and Lipidomics are Affected by Sex Hormones}

Steatosis will invariably occur as a result of an imbalance among enhanced steatogenesis and decreased capacity of oxidation of fatty acids [170]. Moreover, qualitative changes in the hepatic lipidomics may concur with lipotoxicity. Data suggest that all these mechanisms are under the control of sex hormones. For example ovariectomy in rats is associated with increased intrahepatic steatogenesis which occurs through a decreased synthesis of peroxisome proliferator-activated receptor (PPAR), and an increased transcription of genes encoding sterol regulatory element-binding protein 1 [(SREBP-1), a nuclear transcription factor and master regulator of the endogenous synthesis of cholesterol, fatty acids, triglycerides, and phospholipids] and stearoyl coenzyme A desaturase 1 [(SCD1), the rate-limiting enzyme for generating monounsaturated fatty acids (MUFA) from saturated fatty acids protects from hepatocyte lipotoxicity]; all these effects of 


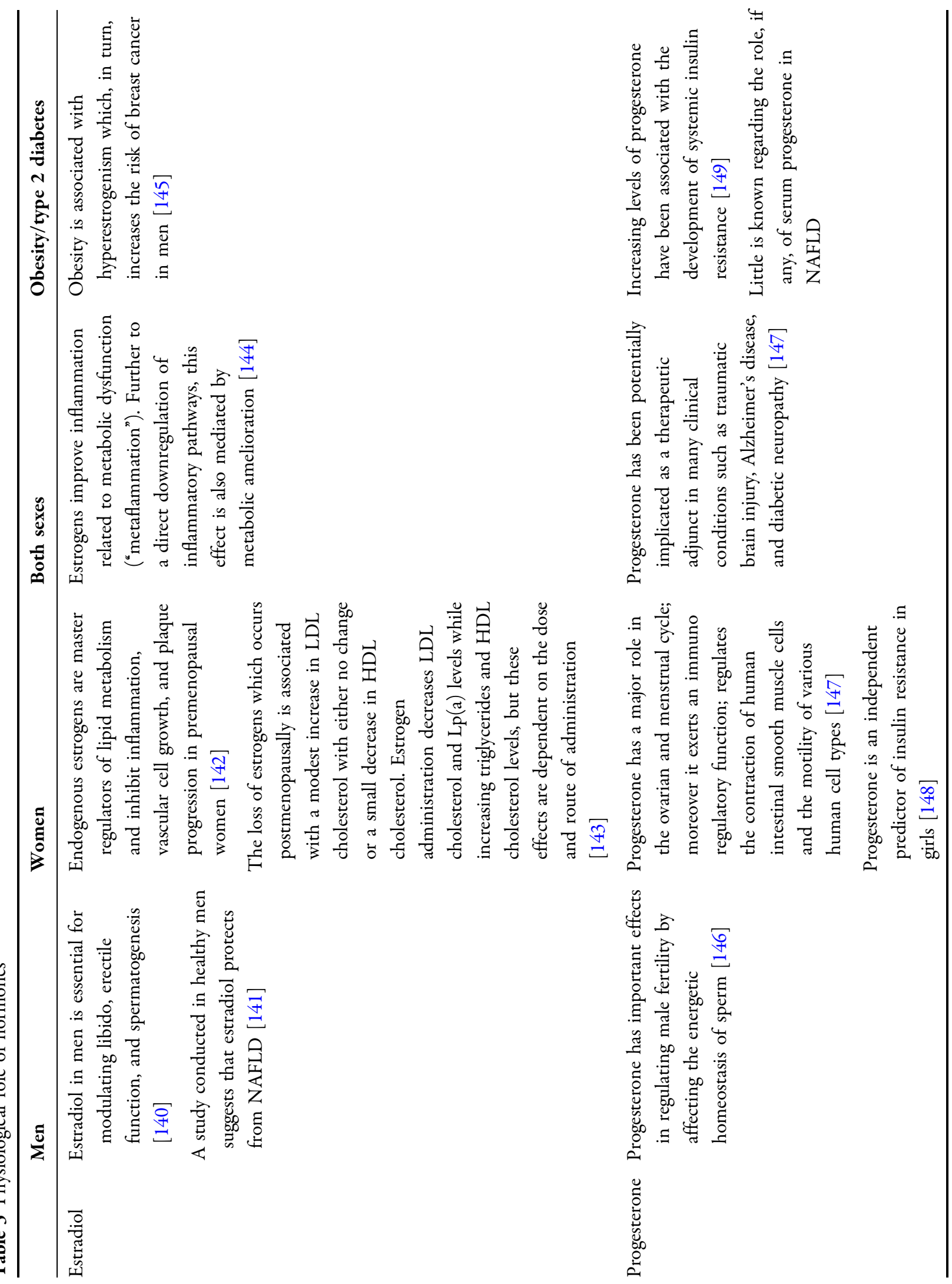




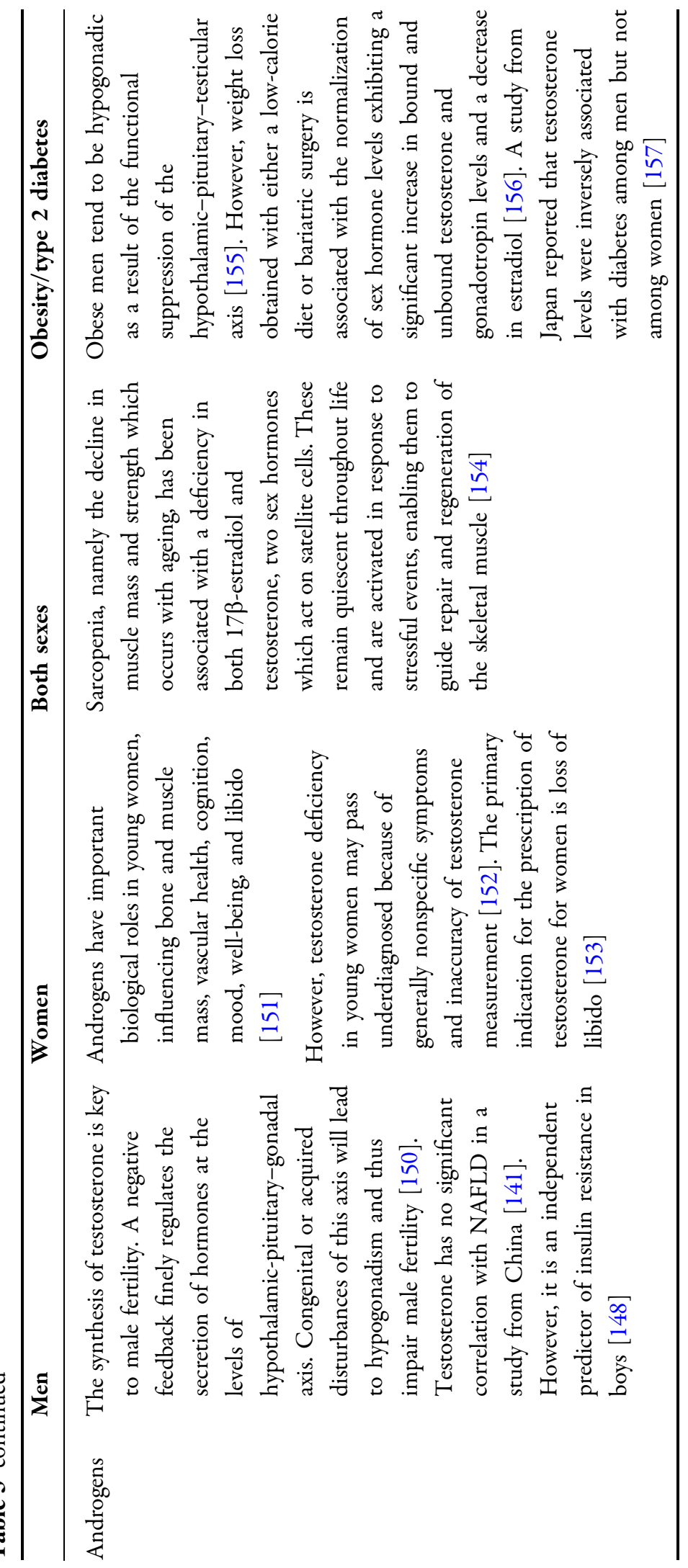


ovariectomy are prevented by $177 \beta$-estradiol replacement, indicating a role for estrogens in the prevention of hepatic fat accumulation [171]. Consistently, hypoestrogenemia is associated with hepatic steatosis through changes in gene expression of molecules related to fat oxidation and lipogenesis; resistance and endurance training prevent this both in rats and human models [172-175]. Moreover, a normal activity of stearoyl-CoA desaturase (SCD), the rate-limiting enzyme for generating monounsaturated fatty acids from saturated fatty acids, protects from hepatocyte lipotoxicity. Indeed, in mouse models of NAFLD, either genetic manipulation or dietary changes that inhibit the activity of SCD promote fibrosing NASH via hepatocyte lipotoxicity, despite inhibiting obesity and improving insulin resistance $[176,177]$. Interestingly, ovarian hormones are also involved in MUFA biosynthesis, via SCD1 [178].

Excess 16:0 fatty acids associated with de novo lipogenesis from high carbohydrate diet inhibits the synthesis of highly unsaturated fatty acids; this may potentially account for the improved metabolic profile which results from supplementing a hypercaloric diet with preformed eicosapentaenoic acid (EPA) and docosahexaenoic acid (DHA) [179]. For example, findings from a genetically engineered NASH mouse model fed a Western diet have shown that dietary DHA was superior to EPA in attenuating Western diet-induced hyperlipidemia and hepatic injury and at reversing those deleterious metabolic and histological effects induced in the liver by a Western diet [180]. Interestingly, human (women using the contraceptive pill or HRT and transsexual subjects) and experimental data in rats consistently indicate that sex hormones act to modify plasma and tissue n-3 PUFA content, possibly by altering the expression of desaturase and elongase enzymes in the liver [181]. Collectively, these findings suggest that enzyme activities which are key in the development of a potentially hepatotoxic lipidomic signature are critically controlled by sex hormones. Finally, it is of clinical importance that the therapeutic activity of PPAR- $\alpha$ agonists in obesity and fatty acid oxidation is modulated by sex and estrogens [182].

\section{Oxidative Stress and Antioxidant Mechanisms}

Reactive oxygen species (ROS) result from the oxidation of fatty acids within mitochondria and peroxisomes. The antioxidant defenses which physiologically constrain oxidative stress are hindered by nutritional deficiencies or changes in the intestinal microbiome that limit availability of choline [183]; by aging and the content of cysteine in diet, which, in turn, will affect the intrahepatic synthesis of glutathione [184]; and by sex and menopause which both affect the normal metabolism of choline [185].

\section{Endotoxin}

Quali-quantitative changes in intestinal microbiota (dysbiosis), which are often associated with dietary indiscretions, have consistently been associated with increased gut permeability which could lead to increased translocation of bacterial products from the intestinal lumen into the portal circulation, thereby triggering chronic inflammation [170]. It this context, it is relevant that in an experimental model of liver failure due to endotoxemia after hepatectomy, sexually mature female rats are more exposed than males to endotoxin-induced liver injury and that ovariectomy abrogates this sexual dimorphism [186]. Whether this also applies to human NAFLD, however, remains to be proven.

\section{Interindividual Variation in Immune Responses is Another Key Liver Disease Modifier in NAFLD}

Whether metabolic inflammation is a gender-dimorphic phenomenon has not been explored in a systematic and organized manner. Obesity, however, will typically exhibit changes in the innate and adaptive immune mechanisms [187]. However, how mechanistically such obesity-related dysregulation of immune defenses will impact on the pathogenesis of NASH is not fully elucidated. Experimental data obtained in the $o b / o b$ mouse model suggest a role for natural killer T cells (NKT cells) which may at least partly account for the 
interindividual variation in immune responses being a key modifier in the development and progression of NAFLD [125]. In this respect, it should be highlighted that sex is a major determinant in the immune response. Supporting this notion, one study in young children reported that immune responses to vaccines were consistently higher or equivalent in girls compared with boys [188].

\section{Liver Fibrosis}

Liver fibrosis is the end-stage result of various liver injuries. In recent times, importance has been given to the Hedgehog signaling pathway on the grounds that activation of this pathway will stimulate the proliferation and differentiation of hepatic stellate cells (HSCs) as myofibroblasts (MF-HSCs), and, conversely, inhibiting Hedgehog activity in myofibroblasts derived from HSCs causes them to revert to a more quiescent (namely less fibrogenic) phenotype [125]. Accordingly, Hedgehog ligands and other factors that control fate decisions in HSCs are critical determinants of the development of cirrhosis due to various causes as well of the course of NASH [125]. For example, hepatic expression of Hedgehog ligands and Hedgehog pathway activity progressively increase from simple steatosis, to NASH, and reach highest levels in NASH-cirrhosis [189]. Of major significance to the pathogenesis of NAFLD, the Hedgehog pathway is strongly regulated by lipids [190], and conversely, Hedgehog signaling is a master regulator of body fat distribution and glucose metabolism [191, 192]. These findings suggest that interindividual variation in Hedgehog signaling might contribute to variability in both hepatic and extrahepatic outcomes of the metabolic syndrome [125].

Experimental overexpression of Hedgehog ligand in hepatocytes is able to induce a fibrogenic liver response and to promote hepatocarcinogenesis in a transgenic mouse model [193]. At least in part by modulating intermediary metabolism [194, 195], Hedgehog also interacts with pathways which regulate growth and differentiation [194, 196-198], such as Wnt/ $\beta$-catenin signaling, which are of potential significance for the development of hepatocellular carcinoma [199].

Treatment with compounds, e.g., vitamin E, that suppress Hedgehog ligand production and reduce the hepatic accumulation of Hedgehog-responsive myofibroblasts has proven beneficial in human NASH [200].

Further studies are necessary to establish whether and to what extent sex hormones affect Hedgehog signaling and how manipulation of cellular energy homeostasis might be exploited to prevent and manage fibrosing NASH, cirrhosis, and HCC in individuals with NAFLD.

\section{CAN GENDER DIMORPHISM OF NAFLD BE EXPLOITED FOR THERAPEUTIC PURPOSES?}

In principle, sex differences found in NAFLD may be accounted for by the effects of sex chromosomes; sex hormones; and by differences in dietary and lifestyle habits [201, 202]. A better understanding of the physiopathological peculiarities of NAFLD in women may contribute to developing tailored therapeutic interventions [203]. Accordingly, the roles of estrogen and HRT, the metabolism of choline, and the effect of weight reduction and exercising in women are discussed in detail hereafter.

\section{Estrogens}

As detailed in Table 3, estrogens exert several beneficial metabolic effects. Experimental studies suggest that estrogens promote the accumulation of peripheral gluteofemoral subcutaneous adipose tissue and, within the liver, promote FFA beta-oxidation and prevent the accumulation of triglycerides; moreover, they regulate energy homeostasis, enhance insulin sensitivity, and exert a protective role on the function of pancreatic beta-cells [201]. Finally, estrogens seem to have antisteatotic, antioxidant, and antifibrogenic properties in the liver [204, 205]. Estrogens may protect from liver steatosis and fibrosis in female mice via upregulation of miRNA-125b and miRNA-29, 
respectively [206, 207]. A recent experimental study showed that the estrogen/estrogen receptor alpha signaling plays essential roles in ROS detoxification and in the reduction of oxidative damage in the liver via partnering with hepatic PPARG coactivator 1 alpha, thus halting the transition from simple steatosis to steatohepatitis [205]. Estrogens are also known to inhibit stellate cell activation and fibrogenesis in experimental models [204]. Of note, a study showed that the natural estrogen $17 \beta$-estradiol prevents deoxycholic acid-induced hepatocellular damage in several cell lines. This hepatoprotective effect of estrogen was sustained by mechanisms which were unlikely to be mediated by nuclear estrogen receptor alpha [208]. Interestingly, it has recently been demonstrated that a non-nuclear estrogen receptor plays a key role in reducing hepatic steatosis in female mice [209]. Similarly, estrogen deficiency leads to fat redistribution toward visceral fat accumulation and its inherently unfavorable metabolic derangements, and is thus able to induce the development and progression of NAFLD/NASH both in mice and humans. Of note, both aromatase knockout mice, which are unable to synthesize endogenous estrogen, and estrogen receptor alpha knockout mice develop hepatic steatosis [210-212]. Patients with breast cancer treated with tamoxifen, a potent estrogen antagonist, develop progressive liver steatosis and NASH [213]. Surgical and physiological menopausal status have been invariably associated with excess risk of NAFLD progression and liver fibrosis, and duration of estrogen deficiency has been directly related to increased fibrosis risk in postmenopausal women with NAFLD $[95,96,98,136]$.

\section{Hormonal Replacement Therapy}

Despite the above premises, the role of HRT in reverting the metabolic alterations associated with low levels of estrogens is a matter of dispute. In animal models, both estrogen and raloxifene, a second-generation selective estrogen receptor modulator, have improved inflammation and ballooning so halting the progression of liver fibrosis in diet-induced NASH in female ovariectomized mice [30, 214]. However, the theoretical benefits of HRT in liver disease remain to be proven in humans. A seminal randomized placebo-controlled study suggested that HRT containing low-dose estradiol and norethisterone was able to reduce liver enzyme concentrations in women with T2D, potentially through the reduction of accumulation of fat in the liver [45]. Accordingly, a north American study using data of the NHANES III survey showed that postmenopausal women who received HRT had a significantly lower risk of NAFLD than postmenopausal women who did not (OR 0.69, 95\% CI 0.48-0.99) [215]. However, whether HRT reduces the risk of NASH and/or fibrosis among postmenopausal women remains uncertain. Two studies showed no, or borderline, beneficial effects of HRT on fibrosis among postmenopausal women with NAFLD [95, 96]. Worryingly, a recent study reported that synthetic hormone use was associated with more severe hepatocellular injury and lobular inflammation. Further analysis showed that progesterone, but not estrogen use, was associated with worse liver histological lesions, suggesting a multifaceted impact of sex hormones on NASH features [97].

\section{Choline}

Several lines of evidence support a key role of choline as an essential nutrient and cellular component. Choline is the major source of methyl groups in the diet and is a precursor of phosphatidylcholine, which is an important component of cell membranes and VLDL, required for the export of lipids from the liver into the bloodstream. Thus, depletion of choline inhibits hepatic triglyceride export and induces fatty liver in both experimental models and humans [185, 203, 216, 217]. Of note, postmenopausal women are more prone than premenopausal women and men to develop NAFLD as a consequence of choline deficiency $[128,185,217]$, and decreased dietary choline intake has been significantly associated with increased fibrosis in postmenopausal women 
with NAFLD [218]. Indeed, menopausal status determines a differential susceptibility to choline deficiency arising from the estrogen-inducible expression and activity of phosphatidylethanolamine- $N$-methyltransferase

(PEMT), a key enzyme in de novo choline biosynthetic pathway. Following menopause, the endogenous supply of choline decreases as a result of estrogen deficiency; consequently, the dietary requirement of choline is increased $[185,203,216,217]$. Consistently, choline supplementation has been reported to improve or revert liver steatosis in patients receiving long-term total parenteral nutrition [219]. However, no interventional studies have addressed whether choline supplementation is able to prevent NAFLD or reduce NAFLD progression; moreover, future studies investigating the role of preventive or therapeutic choline supplementation in postmenopausal women are eagerly awaited.

\section{Weight Reduction and Exercise}

Lifestyle changes, i.e., physical activity and balanced diet, are the milestone of intervention for NAFLD treatment. In general, weight loss induced by lifestyle changes improves IR and features of the MetS, including NAFLD. Of note, a modest weight loss of as little as $2-3 \mathrm{~kg}$ is associated with NAFLD reversal [39], and weight loss of greater than 7-10\% significantly improves histological features of NASH, including fibrosis [220]. However, significant weight reduction achieved through dietary restrictions results in negative effects on lean muscle and bone mass in postmenopausal women. Therefore, while weight reduction in postmenopausal women with metabolic abnormalities and risk factors for NAFLD should be strongly recommended, special attention should be paid to how weight loss is obtained in older women to prevent the worsening of loss of lean mass $[139,203]$. An integrated approach consisting of dietary changes along with regular exercise is mandatory to prevent the untoward effect of losing lean mass. Both resistance training and aerobic exercise are effective in preventing muscle and bone loss during weight loss [203], and equally reduce liver fat content [221, 222]. Postmenopausal women with higher levels of physical activity seem to have lower total body and visceral fat and to be less likely to gain fat mass during menopause $[139,223]$. However, only few studies have specifically assessed the role of physical activity in NAFLD prevention/reversal in postmenopausal women. Data have shown that exercise training effectively reduces liver enzymes in overweight postmenopausal women, probably as a reflection of liver fat content [224], and aerobic physical activity reduces cardiovascular risk factors in postmenopausal women with NAFLD [225]. Further studies are needed to define the ideal structure and duration of exercise-based interventions in postmenopausal women with NAFLD or at risk of developing it.

In summary, there are no codified therapeutic interventions approved for tackling NAFLD in women. Given the theoretical beneficial metabolic and hepatic effects of estrogens, it is intriguing to speculate that HRT might possibly play a role in the prevention and treatment of metabolic alterations associated with menopause, including NAFLD. However, studies in humans are lacking and the potential metabolic benefits should be weighed, in clinical practice, against the detrimental cardiovascular and neoplastic risk associated with long-term HRT [226, 227]. Moreover, the multifaceted and variable impact of different sex hormones in NAFLD should be carefully considered. Dietary changes associated with either aerobic or resistance physical exercise should be considered as the milestone of treatment of NAFLD. Future studies will have to evaluate the effects on NAFLD prevention/treatment of supplementing choline to a balanced diet in postmenopausal women. Finally, the physiopathological peculiarities of NAFLD in women and the gender-based differences in the kinetics and dynamics of drugs and xenobiotics should be taken into account when developing new treatment strategies and proposing interventional trials for NAFLD.

\section{CONCLUSION}

Consistent with the notion that NAFLD and $\mathrm{NASH}$ are strongly linked with hormonal 


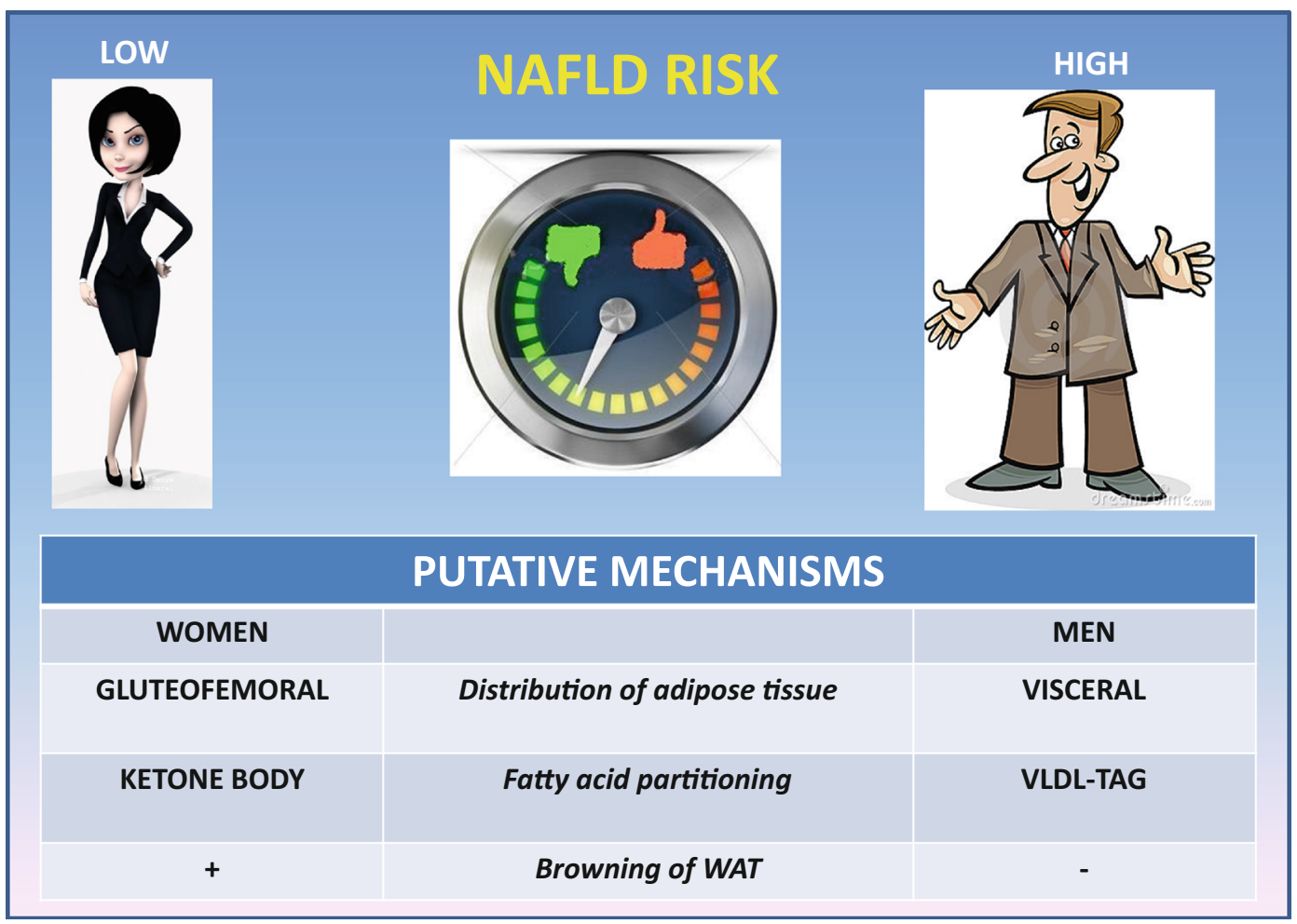

Fig. 1 Physiopathological grounds accounting for male sex as a strong predictor of NAFLD. Male gender and menopausal status have been associated with the risk of NAFLD independently of age and metabolic factors in cross-sectional studies. On the basis of human studies and extrapolation of notions from animal studies, it can be speculated that female sex is protected from dysmetabolism thanks to young individuals' ability to partition fatty acids towards ketone body production rather than VLDL-TAG

influences [228], this narrative review article was driven by the hypothesis that what we called the "sexual dimorphism" of NAFLD might be useful in identifying clues of pathogenic significance and providing hints for prevention and treatment of NAFLD. On these grounds, a systematic research of the literature was conducted. What we found was that not only are men at an increased risk of developing NAFLD (Fig. 1) but also significant age-related changes in NAFLD epidemiology in women may potentially bear physiopathological, clinical, and therapeutic significance. NAFLD epidemiology and physiopathology are modulated by age at menarche and postmenopausal status (Fig. 2). It would be expected that early menarche, definitely associated with estrogen
[23], and to sex-specific browning of white adipose tissue which contributes in protecting female mice from experimental NAFLD associated with methionine choline deficient diet [24]. However, after menopause women display a similar or even higher prevalence of NAFLD compared to men, supporting a protective effect of estrogens. Finally, risk factors associated with NAFLD development are different in men compared to women. $T A G$ triacylglycerols, WAT white adipose tissue

activation, would produce protection against the risk of NAFLD. Nevertheless, it has been suggested that early menarche may confer an increased risk of NAFLD in adulthood, excess adiposity being the primary culprit of this association. Fertile age may be associated with more severe hepatocyte injury and inflammation, but also with a decreased risk of liver fibrosis compared to men and postmenopausal status. Ovarian senescence is strongly associated with severe steatosis and fibrosing NASH which may occur in postmenopausal women. Estrogen deficiency is deemed to be responsible for these findings via the development of postmenopausal metabolic syndrome. Estrogen supplementation may at least theoretically protect from NAFLD development 


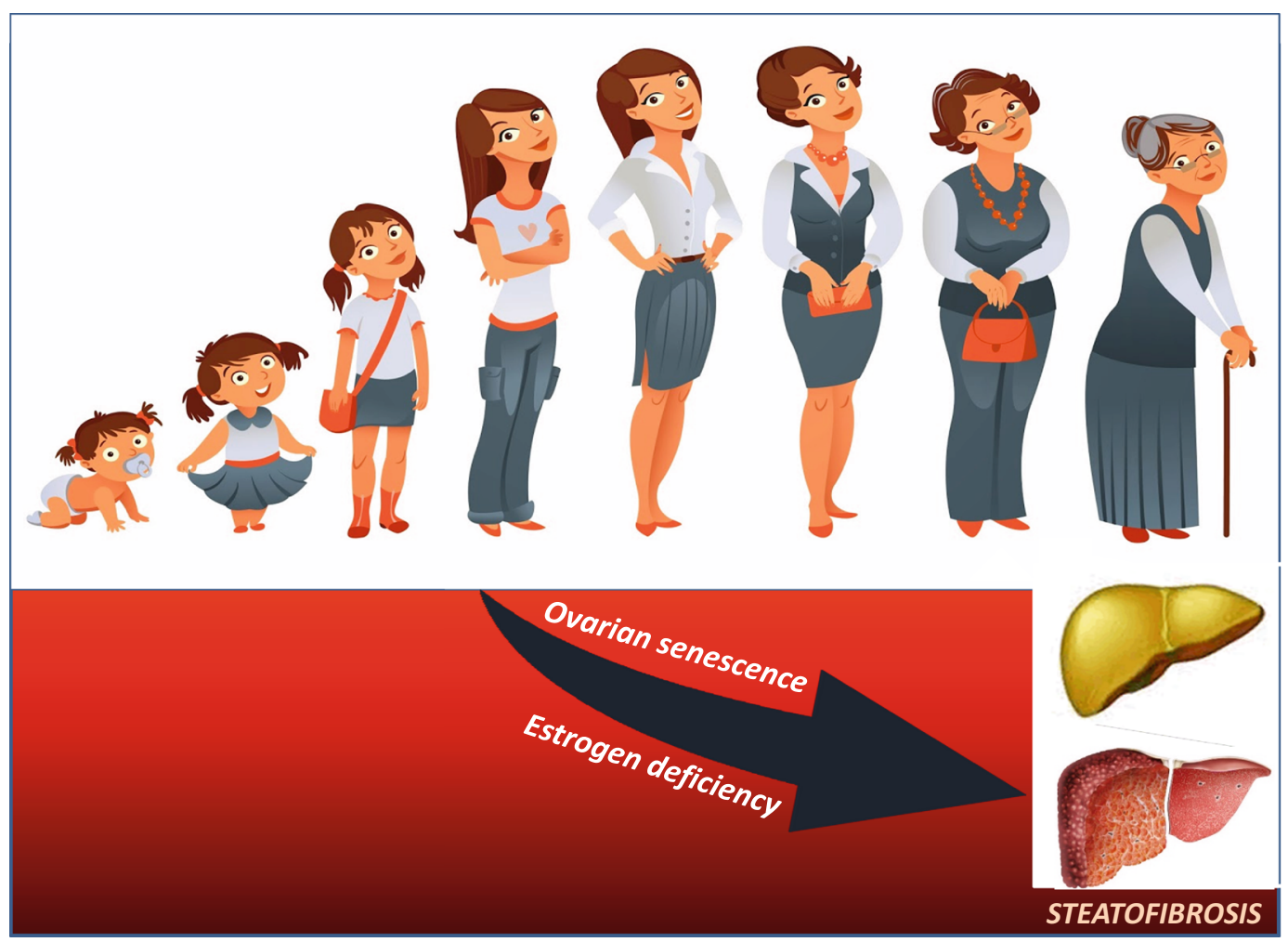

Fig. 2 Hormonal changes are a major determinant of progressive NAFLD in human menopause. NAFLD epidemiology and physiopathology are modulated by age at menarche and postmenopausal status. For example, early menarche may confer an increased risk of NAFLD in adulthood partly mediated by excess adiposity

and progression, as suggested by some studies exploring the effect of HRT on postmenopausal women, but the variable impact of different sex hormones in NAFLD (i.e., the pro-inflammatory effect of progesterone) should be carefully taken into account when proposing treatment with synthetic hormones.

Taken collectively, these data may generate innovative hypotheses to be tested in appropriate clinical and experimental studies on NAFLD physiopathology and treatment.

\section{ACKNOWLEDGEMENTS}

No funding or sponsorship was received for this study or publication of this article.
[130, 131, 133]. Moreover, ovarian senescence, via estrogen deficiency, may eventually be conducive to both massive liver steatosis and its fibrotic evolution via dysmetabolic traits including T2D, hypertriglyceridemia, and visceral obesity which are often found postmenopausally $[29,81,135]$

All named authors meet the International Committee of Medical Journal Editors (ICMJE) criteria for authorship for this manuscript, take responsibility for the integrity of the work as a whole, and have given final approval for the version to be published.

Stefano Ballestri, Fabio Nascimbeni, and Amedeo Lonardo conceived the idea of this article and wrote its first draft. All the authors have read, edited, and approved the various versions of this article.

Disclosures. Stefano Ballestri and Enrica Baldelli have nothing to disclose. Fabio Nascimbeni is involved as a researcher in the "Phase 3, Double Blind, Randomized, Long-Term, Placebo-controlled, Multicenter 
study evaluating the Safety and Efficacy of Obethicolic Acid in Subjects with NASH" (EudraCT 2015-002560-16). Alessandra Marrazzo is involved as a researcher in the "Phase 3 , Double Blind, Randomized, Long-Term, Placebo-controlled, Multicenter study evaluating the Safety and Efficacy of Obethicolic Acid in Subjects with NASH" (EudraCT 2015-002560-16). Amedeo Lonardo is involved as a researcher in the "Phase 3, Double Blind, Randomized, Long-Term, Placebo-controlled, Multicenter study evaluating the Safety and Efficacy of Obethicolic Acid in Subjects with NASH" (EudraCT 2015-002560-16). Dante Romagnoli serves as a consultant for AbbVie.

Compliance with Ethics Guidelines. This article is based on previously conducted studies and does not involve any new studies of human or animal subjects performed by any of the authors.

Data Availability. All data generated or analyzed during this study are included in this published article.

Open Access. This article is distributed under the terms of the Creative Commons Attribution-NonCommercial 4.0 International License (http://creativecommons.org/licenses/ by-nc/4.0/), which permits any noncommercial use, distribution, and reproduction in any medium, provided you give appropriate credit to the original author(s) and the source, provide a link to the Creative Commons license, and indicate if changes were made.

\section{REFERENCES}

1. Brunt EM. Nonalcoholic fatty liver disease: pros and cons of histologic systems of evaluation. Int J Mol Sci 2016;17.

2. Ballestri S, Nascimbeni F, Romagnoli D, et al. The role of nuclear receptors in the pathophysiology, natural course, and drug treatment of NAFLD in humans. Adv Ther. 2016;33:291-319.

3. Piscaglia F, Svegliati-Baroni G, Barchetti A, et al. Clinical patterns of hepatocellular carcinoma in nonalcoholic fatty liver disease: a multicenter prospective study. Hepatology. 2016;63:827-38.

4. Petta S, Valenti L, Bugianesi E, et al. A "systems medicine" approach to the study of non-alcoholic fatty liver disease. Dig Liver Dis. 2016;48:333-42.

5. Byrne CD, Targher G. NAFLD: a multisystem disease. J Hepatol. 2015;62:S47-64.

6. Lonardo A, Sookoian S, Chonchol M, et al. Cardiovascular and systemic risk in nonalcoholic fatty liver disease-atherosclerosis as a major player in the natural course of NAFLD. Curr Pharm Des. 2013;19:5177-92.

7. Loria P, Marchesini G, Nascimbeni F, et al. Cardiovascular risk, lipidemic phenotype and steatosis. A comparative analysis of cirrhotic and non-cirrhotic liver disease due to varying etiology. Atherosclerosis. 2014;232:99-109.

8. Lonardo A, Ballestri S, Targher G, et al. Diagnosis and management of cardiovascular risk in nonalcoholic fatty liver disease. Expert Rev Gastroenterol Hepatol. 2015;9:629-50.

9. Targher G, Byrne CD, Lonardo A, et al. Non-alcoholic fatty liver disease and risk of incident cardiovascular disease: a meta-analysis. J Hepatol. 2016;65:589-600.

10. Nascimbeni F, Pais R, Bellentani $S$, et al. From NAFLD in clinical practice to answers from guidelines. J Hepatol. 2013;59:859-71.

11. Lonardo A, Bellentani S, Ratziu V, et al. Insulin resistance in nonalcoholic steatohepatitis: necessary but not sufficient - death of a dogma from analysis of therapeutic studies? Expert Rev Gastroenterol Hepatol. 2011;5:279-89.

12. Ballestri S, Nascimbeni F, Romagnoli D, et al. The independent predictors of non-alcoholic steatohepatitis and its individual histological features. Insulin resistance, serum uric acid, metabolic syndrome, alanine aminotransferase and serum total cholesterol are a clue to pathogenesis and candidate targets for treatment. Hepatol Res. 2016;46:1074-87.

13. Ballestri S, Zona S, Targher G, et al. Nonalcoholic fatty liver disease is associated with an almost twofold increased risk of incident type 2 diabetes and metabolic syndrome. Evidence from a systematic review and meta-analysis. J Gastroenterol Hepatol. 2016;31:936-44.

14. Younossi ZM, Koenig AB, Abdelatif D, et al. Global epidemiology of nonalcoholic fatty liver diseasemeta-analytic assessment of prevalence, incidence, and outcomes. Hepatology. 2016;64:73-84. 
15. Non-Alcoholic Fatty Liver Disease Study Group, Lonardo A, Bellentani S, et al. Epidemiological modifiers of non-alcoholic fatty liver disease: focus on high-risk groups. Dig Liver Dis. 2015;47:997-1006.

16. Baumeister SE, Volzke H, Marschall P, et al. Impact of fatty liver disease on health care utilization and costs in a general population: a 5-year observation. Gastroenterology. 2008;134:85-94.

17. Loria P, Lonardo A, Anania F. Liver and diabetes. A vicious circle. Hepatol Res. 2013;43:51-64.

18. Lonardo A, Sookoian S, Pirola CJ, et al. Non-alcoholic fatty liver disease and risk of cardiovascular disease. Metabolism. 2016;65:1136-50.

19. Younossi ZM, Blissett D, Blissett R, et al. The economic and clinical burden of nonalcoholic fatty liver disease in the United States and Europe. Hepatology. 2016;64:1577-86.

20. Day CP. Pathogenesis of steatohepatitis. Best Pract Res Clin Gastroenterol. 2002;16:663-78.

21. Tilg H, Moschen AR. Evolution of inflammation in nonalcoholic fatty liver disease: the multiple parallel hits hypothesis. Hepatology. 2010;52:1836-46.

22. Day CP, James OF. Steatohepatitis: a tale of two "hits"? Gastroenterology. 1998;114:842-5.

23. Marinou K, Adiels M, Hodson L, et al. Young women partition fatty acids towards ketone body production rather than VLDL-TAG synthesis, compared with young men. Br J Nutr. 2011;105:857-65.

24. Lee YH, Kim SH, Kim SN, et al. Sex-specific metabolic interactions between liver and adipose tissue in MCD diet-induced non-alcoholic fatty liver disease. Oncotarget. 2016;7:46959-46971.

25. Ludwig J, Viggiano TR, McGill DB, et al. Nonalcoholic steatohepatitis: Mayo Clinic experiences with a hitherto unnamed disease. Mayo Clin Proc. 1980;55:434-8.

26. Lonardo A, Carani C, Carulli N, et al. 'Endocrine NAFLD' a hormonocentric perspective of nonalcoholic fatty liver disease pathogenesis. J Hepatol. 2006;44:1196-207.

27. Wenger NK. Coronary heart disease: an older woman's major health risk. BMJ. 1997;315:1085-90.

28. Fadini GP, de Kreutzenberg S, Albiero M, et al. Gender differences in endothelial progenitor cells and cardiovascular risk profile: the role of female estrogens. Arterioscler Thromb Vasc Biol. 2008;28:997-1004.
29. Turola E, Petta S, Vanni E, et al. Ovarian senescence increases liver fibrosis in humans and zebrafish with steatosis. Dis Model Mech. 2015;8:1037-46.

30. Kamada Y, Kiso S, Yoshida Y, et al. Estrogen deficiency worsens steatohepatitis in mice fed high-fat and high-cholesterol diet. Am J Physiol Gastrointest Liver Physiol. 2011;301:G1031-43.

31. Brady CW. Liver disease in menopause. World J Gastroenterol. 2015;21:7613-20.

32. Targher G, Rossini M, Lonardo A. Evidence that non-alcoholic fatty liver disease and polycystic ovary syndrome are associated by necessity rather than chance: a novel hepato-ovarian axis? Endocrine. 2016;51:211-21.

33. Kojima S, Watanabe N, Numata $M$, et al. Increase in the prevalence of fatty liver in Japan over the past 12 years: analysis of clinical background. J Gastroenterol. 2003;38:954-61.

34. Hamaguchi M, Kojima T, Takeda N, et al. The metabolic syndrome as a predictor of nonalcoholic fatty liver disease. Ann Intern Med. 2005;143:722-8.

35. Suzuki A, Angulo P, Lymp J, et al. Chronological development of elevated aminotransferases in a nonalcoholic population. Hepatology. 2005;41:64-71.

36. Tsuneto A, Hida A, Sera N, et al. Fatty liver incidence and predictive variables. Hypertens Res. 2010;33:638-43.

37. Hamaguchi M, Kojima T, Ohbora A, et al. Aging is a risk factor of nonalcoholic fatty liver disease in premenopausal women. World J Gastroenterol. 2012;18:237-43.

38. Zhou YJ, Li YY, Nie YQ, et al. Natural course of nonalcoholic fatty liver disease in southern China: a prospective cohort study. J Dig Dis. 2012;13:153-60.

39. Zelber-Sagi S, Lotan R, Shlomai A, et al. Predictors for incidence and remission of NAFLD in the general population during a seven-year prospective follow-up. J Hepatol. 2012;56:1145-51.

40. Sung KC, Kim BS, Cho YK, et al. Predicting incident fatty liver using simple cardio-metabolic risk factors at baseline. BMC Gastroenterol. 2012;12:84.

41. Xu C, Yu C, Ma H, et al. Prevalence and risk factors for the development of nonalcoholic fatty liver disease in a nonobese Chinese population: the Zhejiang Zhenhai Study. Am J Gastroenterol. 2013;108:1299-304. 
42. Wong VW, Wong GL, Yeung DK, et al. Incidence of non-alcoholic fatty liver disease in Hong Kong: a population study with paired proton-magnetic resonance spectroscopy. J Hepatol. 2015;62:182-9.

43. Yun KE, Nam GE, Lim J, et al. Waist gain is associated with a higher incidence of nonalcoholic fatty liver disease in Korean adults: a cohort study. PLoS One. 2016;11:e0158710.

44. Bruno S, Maisonneuve P, Castellana P, et al. Incidence and risk factors for non-alcoholic steatohepatitis: prospective study of 5408 women enrolled in Italian tamoxifen chemoprevention trial. BMJ. 2005;330:932.

45. McKenzie J, Fisher BM, Jaap AJ, et al. Effects of HRT on liver enzyme levels in women with type 2 diabetes: a randomized placebo-controlled trial. Clin Endocrinol (Oxf). 2006;65:40-4.

46. Shen L, Fan JG, Shao Y, et al. Prevalence of nonalcoholic fatty liver among administrative officers in Shanghai: an epidemiological survey. World J Gastroenterol. 2003;9:1106-10.

47. Fan JG, Zhu J, Li XJ, et al. Prevalence of and risk factors for fatty liver in a general population of Shanghai, China. J Hepatol. 2005;43:508-14.

48. Chen $\mathrm{CH}$, Huang $\mathrm{MH}$, Yang JC, et al. Prevalence and risk factors of nonalcoholic fatty liver disease in an adult population of Taiwan: metabolic significance of nonalcoholic fatty liver disease in nonobese adults. J Clin Gastroenterol. 2006;40:745-52.

49. Park SH, Jeon WK, Kim SH, et al. Prevalence and risk factors of non-alcoholic fatty liver disease among Korean adults. J Gastroenterol Hepatol. 2006;21:138-43.

50. Zelber-Sagi S, Nitzan-Kaluski D, Halpern Z, et al. Prevalence of primary non-alcoholic fatty liver disease in a population-based study and its association with biochemical and anthropometric measures. Liver Int. 2006;26:856-63.

51. Zhou YJ, Li YY, Nie YQ, et al. Prevalence of fatty liver disease and its risk factors in the population of South China. World J Gastroenterol. 2007;13:6419-24.

52. Li H, Wang YJ, Tan K, et al. Prevalence and risk factors of fatty liver disease in Chengdu, Southwest China. Hepatobiliary Pancreat Dis Int. 2009;8:377-82.

53. Caballeria L, Pera G, Auladell MA, et al. Prevalence and factors associated with the presence of nonalcoholic fatty liver disease in an adult population in Spain. Eur J Gastroenterol Hepatol. 2010;22:24-32.
54. Hu X, Huang Y, Bao Z, et al. Prevalence and factors associated with nonalcoholic fatty liver disease in Shanghai work-units. BMC Gastroenterol. 2012;12:123.

55. Wong VW, Chu WC, Wong GL, et al. Prevalence of non-alcoholic fatty liver disease and advanced fibrosis in Hong Kong Chinese: a population study using proton-magnetic resonance spectroscopy and transient elastography. Gut. 2012;61:409-15.

56. Eguchi Y, Hyogo H, Ono M, et al. Prevalence and associated metabolic factors of nonalcoholic fatty liver disease in the general population from 2009 to 2010 in Japan: a multicenter large retrospective study. J Gastroenterol. 2012;47:586-95.

57. Younossi ZM, Stepanova M, Negro F, et al. Nonalcoholic fatty liver disease in lean individuals in the United States. Medicine (Baltimore). 2012;91:319-27.

58. Lazo M, Hernaez R, Eberhardt MS, et al. Prevalence of nonalcoholic fatty liver disease in the United States: the Third National Health and Nutrition Examination Survey, 1988-1994. Am J Epidemiol. 2013;178:38-45.

59. Wang Z, Xu M, Peng J, et al. Prevalence and associated metabolic factors of fatty liver disease in the elderly. Exp Gerontol. 2013;48:705-9.

60. Yan J, Xie W, Ou WN, et al. Epidemiological survey and risk factor analysis of fatty liver disease of adult residents, Beijing, China. J Gastroenterol Hepatol. 2013;28:1654-9.

61. Chiloiro M, Caruso MG, Cisternino AM, et al. Ultrasound evaluation and correlates of fatty liver disease: a population study in a Mediterranean area. Metab Syndr Relat Disord. 2013;11:349-58.

62. Foster T, Anania FA, Li D, et al. The prevalence and clinical correlates of nonalcoholic fatty liver disease (NAFLD) in African Americans: the multiethnic study of atherosclerosis (MESA). Dig Dis Sci. 2013;58:2392-8.

63. Yang W, Huang H, Wang Y, et al. High red blood cell distribution width is closely associated with nonalcoholic fatty liver disease. Eur J Gastroenterol Hepatol. 2014;26:174-8.

64. Saida T, Fukushima W, Ohfuji S, et al. Effect modification of body mass index and body fat percentage on fatty liver disease in a Japanese population. J Gastroenterol Hepatol. 2014;29:128-36.

65. Wang Z, Xu M, Hu Z, et al. Sex-specific prevalence of fatty liver disease and associated metabolic factors in Wuhan, south central China. Eur J Gastroenterol Hepatol. 2014;26:1015-21. 
66. Schneider AL, Lazo M, Selvin E, et al. Racial differences in nonalcoholic fatty liver disease in the US population. Obesity (Silver Spring). 2014;22:292-9.

67. Xiao SJ, Fu GJ, Lv YL, et al. Prevalence and risk factors of fatty liver disease in young and middle-aged population: one center study in Southwestern China. J Gastroenterol Hepatol. 2014;29:358-64.

68. Martinez-Alvarado Mdel R, Juarez-Rojas JG, Medina-Urrutia AX, et al. Association of fatty liver with cardiovascular risk factors and subclinical atherosclerosis in a Mexican population. Rev Invest Clin. 2014;66:407-14.

69. Liu J, Lin H, Zhang C, et al. Non-alcoholic fatty liver disease associated with gallstones in females rather than males: a longitudinal cohort study in Chinese urban population. BMC Gastroenterol. 2014;14:213.

70. Amirkalali B, Poustchi H, Keyvani H, et al. Prevalence of non-alcoholic fatty liver disease and its predictors in North of Iran. Iran J Public Health. 2014;43:1275-83.

71. Nishioji K, Sumida Y, Kamaguchi M, et al. Prevalence of and risk factors for non-alcoholic fatty liver disease in a non-obese Japanese population, 2011-2012. J Gastroenterol. 2015;50:95-108.

72. Huang $\mathrm{X}, \mathrm{Xu} \mathrm{M}$, Chen $\mathrm{Y}$, et al. Validation of the fatty liver index for nonalcoholic fatty liver disease in middle-aged and elderly Chinese. Medicine (Baltimore). 2015;94:e1682.

73. Fattahi MR, Niknam R, Safarpour A, et al. The prevalence of metabolic syndrome in non-alcoholic fatty liver disease; a population-based study. Middle East J Dig Dis. 2016;8:131-7.

74. Motamed N, Sohrabi M, Ajdarkosh H, et al. Fatty liver index vs waist circumference for predicting non-alcoholic fatty liver disease. World J Gastroenterol. 2016;22:3023-30.

75. Lonardo A, Lombardini S, Scaglioni F, et al. Fatty liver, carotid disease and gallstones: a study of age-related associations. World J Gastroenterol. 2006;12:5826-33.

76. Bertolotti M, Lonardo A, Mussi C, et al. Nonalcoholic fatty liver disease and aging: epidemiology to management. World J Gastroenterol. 2014;20:14185-204.

77. Zhu JZ, Dai YN, Wang YM, et al. Prevalence of nonalcoholic fatty liver disease and economy. Dig Dis Sci. 2015;60:3194-202.

78. Denzer C, Thiere D, Muche R, et al. Gender-specific prevalences of fatty liver in obese children and adolescents: roles of body fat distribution, sex steroids, and insulin resistance. J Clin Endocrinol Metab. 2009;94:3872-81.

79. Carulli L, Lonardo A, Lombardini S, et al. Gender, fatty liver and GGT. Hepatology. 2006;44:278-9.

80. Gutierrez-Grobe Y, Ponciano-Rodriguez G, Ramos $\mathrm{MH}$, et al. Prevalence of non alcoholic fatty liver disease in premenopausal, posmenopausal and polycystic ovary syndrome women. The role of estrogens. Ann Hepatol. 2010;9:402-9.

81. Chung GE, Yim JY, Kim D, et al. The influence of metabolic factors for nonalcoholic fatty liver disease in women. Biomed Res Int. 2015;2015:131528.

82. Wang Z, Xu M, Hu Z, et al. Prevalence of nonalcoholic fatty liver disease and its metabolic risk factors in women of different ages and body mass index. Menopause. 2015;22:667-73.

83. Volzke H, Schwarz S, Baumeister SE, et al. Menopausal status and hepatic steatosis in a general female population. Gut. 2007;56:594-5.

84. Florentino GS, Cotrim HP, Vilar CP, et al. Nonalcoholic fatty liver disease in menopausal women. Arq Gastroenterol. 2013;50:180-5.

85. Ryu S, Suh BS, Chang Y, et al. Menopausal stages and non-alcoholic fatty liver disease in middle-aged women. Eur J Obstet Gynecol Reprod Biol. 2015;190:65-70.

86. Rodrigues MH, Bruno AS, Nahas-Neto J, et al. Evaluation of clinical and inflammatory markers of nonalcoholic fatty liver disease in postmenopausal women with metabolic syndrome. Metab Syndr Relat Disord. 2014;12:330-8.

87. Loomis AK, Kabadi S, Preiss D, et al. Body mass index and risk of nonalcoholic fatty liver disease: two electronic health record prospective studies. J Clin Endocrinol Metab. 2016;101:945-52.

88. Calzadilla Bertot L, Adams LA. The natural course of non-alcoholic fatty liver disease. Int $\mathrm{J}$ Mol Sci 2016;17. doi:10.3390/ijms17050774.

89. Argo CK, Northup PG, Al-Osaimi AM, et al. Systematic review of risk factors for fibrosis progression in non-alcoholic steatohepatitis. J Hepatol. 2009;51:371-9.

90. Singh DK, Sakhuja P, Malhotra V, et al. Independent predictors of steatohepatitis and fibrosis in Asian Indian patients with non-alcoholic steatohepatitis. Dig Dis Sci. 2008;53:1967-76.

91. Hossain N, Afendy A, Stepanova M, et al. Independent predictors of fibrosis in patients with 
nonalcoholic fatty liver disease. Clin Gastroenterol Hepatol 2009;7:1224-9, 1229 e1-2.

92. Al-hamoudi W, El-Sabbah M, Ali S, et al. Epidemiological, clinical, and biochemical characteristics of Saudi patients with nonalcoholic fatty liver disease: a hospital-based study. Ann Saudi Med. 2012;32:288-92.

93. Bambha K, Belt P, Abraham M, et al. Ethnicity and nonalcoholic fatty liver disease. Hepatology. 2012;55:769-80.

94. Tapper EB, Krajewski K, Lai M, et al. Simple non-invasive biomarkers of advanced fibrosis in the evaluation of non-alcoholic fatty liver disease. Gastroenterol Rep (Oxf). 2014;2:276-80.

95. Yang JD, Abdelmalek MF, Pang H, et al. Gender and menopause impact severity of fibrosis among patients with nonalcoholic steatohepatitis. Hepatology. 2014;59:1406-14.

96. Klair JS, Yang JD, Abdelmalek MF, et al. A longer duration of estrogen deficiency increases fibrosis risk among postmenopausal women with nonalcoholic fatty liver disease. Hepatology. 2016;64:85-91.

97. Yang JD, Abdelmalek MF, Guy CD, et al. Patient sex, reproductive status, and synthetic hormone use associate with histologic severity of nonalcoholic steatohepatitis. Clin Gastroenterol Hepatol 2017;15:127-131.e2.

98. Yoneda M, Thomas E, Sumida Y, et al. The influence of menopause on the development of hepatic fibrosis in nonobese women with nonalcoholic fatty liver disease. Hepatology. 2014;60:1792.

99. Bedogni G, Miglioli L, Masutti F, et al. Incidence and natural course of fatty liver in the general population: the Dionysos study. Hepatology. 2007;46:1387-91.

100. Ong JP, Pitts A, Younossi ZM. Increased overall mortality and liver-related mortality in non-alcoholic fatty liver disease. J Hepatol. 2008;49:608-12.

101. Haring R, Wallaschofski $H$, Nauck M, et al. Ultrasonographic hepatic steatosis increases prediction of mortality risk from elevated serum gamma-glutamyl transpeptidase levels. Hepatology. 2009;50:1403-11.

102. Villa E. Role of estrogen in liver cancer. Womens Health (Lond). 2008;4:41-50.

103. Reddy SK, Steel JL, Chen HW, et al. Outcomes of curative treatment for hepatocellular cancer in nonalcoholic steatohepatitis versus hepatitis C and alcoholic liver disease. Hepatology. 2012;55:1809-19.
104. Yasui K, Hashimoto E, Komorizono Y, et al. Characteristics of patients with nonalcoholic steatohepatitis who develop hepatocellular carcinoma. Clin Gastroenterol Hepatol. 2011;9:428-33 (quiz e50).

105. Yang D, Hanna DL, Usher J, et al. Impact of sex on the survival of patients with hepatocellular carcinoma: a surveillance, epidemiology, and end results analysis. Cancer. 2014;120:3707-16.

106. Ballestri S, Lonardo A, Bonapace S, et al. Risk of cardiovascular, cardiac and arrhythmic complications in patients with non-alcoholic fatty liver disease. World J Gastroenterol. 2014;20:1724-45.

107. Mantovani A, Ballestri S, Lonardo A, et al. cardiovascular disease and myocardial abnormalities in nonalcoholic fatty liver disease. Dig Dis Sci. 2016;61:1246-67.

108. Wells GL. Cardiovascular risk factors: does sex matter? Curr Vasc Pharmacol. 2016;14:452-7.

109. Stepanova M, Younossi ZM. Independent association between nonalcoholic fatty liver disease and cardiovascular disease in the US population. Clin Gastroenterol Hepatol. 2012;10:646-50.

110. Treeprasertsuk S, Leverage S, Adams LA, et al. The Framingham risk score and heart disease in nonalcoholic fatty liver disease. Liver Int. 2012;32:945-50.

111. Kim D, Choi SY, Park EH, et al. Nonalcoholic fatty liver disease is associated with coronary artery calcification. Hepatology. 2012;56:605-13.

112. Mirbagheri SA, Rashidi A, Abdi S, et al. Liver: an alarm for the heart? Liver Int. 2007;27:891-4.

113. Acikel M, Sunay S, Koplay M, et al. Evaluation of ultrasonographic fatty liver and severity of coronary atherosclerosis, and obesity in patients undergoing coronary angiography. Anadolu Kardiyol Derg. 2009;9:273-9.

114. Sun L, Lu SZ. Association between non-alcoholic fatty liver disease and coronary artery disease severity. Chin Med J (Engl). 2011;124:867-72.

115. Wong VW, Wong GL, Yip GW, et al. Coronary artery disease and cardiovascular outcomes in patients with non-alcoholic fatty liver disease. Gut. 2011;60:1721-7.

116. Fraser A, Harris R, Sattar N, et al. Gamma-glutamyltransferase is associated with incident vascular events independently of alcohol intake: analysis of the British Women's Heart and Health Study and Meta-Analysis. Arterioscler Thromb Vasc Biol. 2007;27:2729-35. 
117. Ioannou GN, Weiss NS, Boyko EJ, et al. Elevated serum alanine aminotransferase activity and calculated risk of coronary heart disease in the United States. Hepatology. 2006;43:1145-51.

118. Schindhelm RK, Dekker JM, Nijpels G, et al. Alanine aminotransferase predicts coronary heart disease events: a 10-year follow-up of the Hoorn Study. Atherosclerosis. 2007;191:391-6.

119. Feitosa MF, Reiner AP, Wojczynski MK, et al. Sex-influenced association of nonalcoholic fatty liver disease with coronary heart disease. Atherosclerosis. 2013;227:420-4.

120. Buday B, Pach PF, Literati-Nagy B, et al. Sex influenced association of directly measured insulin sensitivity and serum transaminase levels: why alanine aminotransferase only predicts cardiovascular risk in men? Cardiovasc Diabetol. 2015;14:55.

121. Kim HJ, Lim CW, Lee JH, et al. Gender-based differences in the relationship between fatty liver disease and atherosclerosis. Cardiovasc J Afr. 2016;27:281-6.

122. Jepsen P, Vilstrup H, Mellemkjaer L, et al. Prognosis of patients with a diagnosis of fatty liver-a registry-based cohort study. Hepatogastroenterology. 2003;50:2101-4.

123. Hamaguchi M, Kojima T, Takeda N, et al. Nonalcoholic fatty liver disease is a novel predictor of cardiovascular disease. World J Gastroenterol. 2007;13:1579-84.

124. Kim MK, Ahn CW, Nam JS, et al. Association between nonalcoholic fatty liver disease and coronary artery calcification in postmenopausal women. Menopause. 2015;22:1323-7.

125. Suzuki A, Diehl AM. Nonalcoholic steatohepatitis. Annu Rev Med. 2017;68:85-98.

126. Lonardo A, Trande P. Are there any sex differences in fatty liver? A study of glucose metabolism and body fat distribution. J Gastroenterol Hepatol. 2000;15:775-82.

127. Suzuki A, Abdelmalek MF, Unalp-Arida A, et al. Regional anthropometric measures and hepatic fibrosis in patients with nonalcoholic fatty liver disease. Clin Gastroenterol Hepatol. 2010;8:1062-9.

128. Fischer LM, da Costa KA, Kwock L, et al. Dietary choline requirements of women: effects of estrogen and genetic variation. Am J Clin Nutr. 2010;92:1113-9.

129. Bruno Ade S, Rodrigues $\mathrm{MH}$, Alvares $\mathrm{MC}$, et al. Non-alcoholic fatty liver disease and its associated risk factors in Brazilian postmenopausal women. Climacteric. 2014;17:465-71.
130. Lu J, Zhang J, Du R, et al. Age at menarche is associated with the prevalence of NAFLD later in life. J Diabetes. 2017;9:53-60.

131. Mueller NT, Pereira MA, Demerath EW, et al. Earlier menarche is associated with fatty liver and abdominal ectopic fat in midlife, independent of young adult BMI: the CARDIA study. Obesity (Silver Spring). 2015;23:468-74.

132. Laitinen J, Power C, Jarvelin MR. Family social class, maternal body mass index, childhood body mass index, and age at menarche as predictors of adult obesity. Am J Clin Nutr. 2001;74:287-94.

133. Ryu S, Chang Y, Choi Y, et al. Age at menarche and non-alcoholic fatty liver disease. J Hepatol. 2015;62:1164-70.

134. Cao X, Zhou J, Yuan H, et al. Duration of reproductive lifespan and age at menarche in relation to metabolic syndrome in postmenopausal Chinese women. J Obstet Gynaecol Res. 2016;42:1581-7.

135. Kavanagh K, Espeland MA, Sutton-Tyrrell K, et al. Liver fat and SHBG affect insulin resistance in midlife women: the Study of Women's Health Across the Nation (SWAN). Obesity (Silver Spring). 2013;21:1031-8.

136. Matsuo K, Gualtieri MR, Cahoon SS, et al. Surgical menopause and increased risk of nonalcoholic fatty liver disease in endometrial cancer. Menopause. 2016;23:189-96.

137. Codes L, Asselah T, Cazals-Hatem D, et al. Liver fibrosis in women with chronic hepatitis C: evidence for the negative role of the menopause and steatosis and the potential benefit of hormone replacement therapy. Gut. 2007;56:390-5.

138. Lu G, Shimizu I, Cui X, et al. Antioxidant and antiapoptotic activities of idoxifene and estradiol in hepatic fibrosis in rats. Life Sci. 2004;74:897-907.

139. Lavoie JM, Pighon A. NAFLD, estrogens, and physical exercise: the animal model. J Nutr Metab. 2012;2012:914938.

140. Schulster M, Bernie AM, Ramasamy R. The role of estradiol in male reproductive function. Asian J Androl. 2016;18:435-40.

141. Tian GX, Sun Y, Pang CJ, et al. Oestradiol is a protective factor for non-alcoholic fatty liver disease in healthy men. Obes Rev. 2012;13:381-7.

142. Barton M. Cholesterol and atherosclerosis: modulation by oestrogen. Curr Opin Lipidol. 2013;24:214-20.

143. Feingold K, Brinton EA, Grunfeld C. The effect of endocrine disorders on lipids and lipoproteins. In: 
De Groot LJ, Chrousos G, Dungan K, et al, editors. South Dartmouth: Endotext; 2000.

144. Monteiro R, Teixeira D, Calhau C. Estrogen signaling in metabolic inflammation. Mediators Inflamm. 2014;2014:615917.

145. Thomas DB. Breast cancer in men. Epidemiol Rev. 1993;15:220-31.

146. Aquila S, De Amicis F. Steroid receptors and their ligands: effects on male gamete functions. Exp Cell Res. 2014;328:303-13.

147. Taraborrelli S. Physiology, production and action of progesterone. Acta Obstet Gynecol Scand. 2015;94(Suppl 161):8-16.

148. Aldhoon-Hainerova I, Zamrazilova H, Hill M, et al. Insulin sensitivity and its relation to hormones in adolescent boys and girls. Metabolism. 2017;67:90-8.

149. Morita A, Ishigaki Y. Gender-difference in diabetes mellitus. Nihon Rinsho. 2015;73:606-10.

150. Corradi PF, Corradi RB, Greene LW. Physiology of the hypothalamic pituitary gonadal axis in the male. Urol Clin N Am. 2016;43:151-62.

151. Davis SR, Wahlin-Jacobsen S. Testosterone in women-the clinical significance. Lancet Diabetes Endocrinol. 2015;3:980-92.

152. Kalantaridou SN, Calis KA. Testosterone therapy in premenopausal women. Semin Reprod Med. 2006;24:106-14.

153. Davis SR, Worsley R, Miller KK, et al. Androgens and female sexual function and dysfunction-findings from the fourth international consultation of sexual medicine. J Sex Med. 2016;13:168-78.

154. La Colla A, Pronsato L, Milanesi L, et al. 17ß-Estradiol and testosterone in sarcopenia: role of satellite cells. Ageing Res Rev. 2015;24:166-77.

155. Grossmann M. Testosterone and glucose metabolism in men: current concepts and controversies. J Endocrinol. 2014;220:R37-55.

156. Corona G, Rastrelli G, Monami M, et al. Body weight loss reverts obesity-associated hypogonadotropic hypogonadism: a systematic review and meta-analysis. Eur J Endocrinol. 2013;168:829-43.

157. Goto A, Morita A, Goto M, et al. Associations of sex hormone-binding globulin and testosterone with diabetes among men and women (the Saku Diabetes study): a case control study. Cardiovasc Diabetol. 2012;11:130.
158. Dakin RS, Walker BR, Seckl JR, et al. Estrogens protect male mice from obesity complications and influence glucocorticoid metabolism. Int $\mathrm{J}$ Obes (Lond). 2015;39:1539-47.

159. Candia R, Riquelme A, Baudrand R, et al. Overexpression of 11beta-hydroxysteroid dehydrogenase type 1 in visceral adipose tissue and portal hypercortisolism in non-alcoholic fatty liver disease. Liver Int. 2012;32:392-9.

160. Moon SS, Lee YS, Kim JG, et al. Association of 11beta-hydroxysteroid dehydrogenase type 1 gene polymorphisms with serum alanine aminotransferase activity. Diabetes Res Clin Pract. 2013;99:343-50.

161. Stefan N, Ramsauer M, Jordan P, et al. Inhibition of 11beta-HSD1 with RO5093151 for non-alcoholic fatty liver disease: a multicentre, randomised, double-blind, placebo-controlled trial. Lancet Diabetes Endocrinol. 2014;2:406-16.

162. Albiston AL, Smith RE, Krozowski ZS. Sex- and tissue- specific regulation of 11 beta-hydroxysteroid dehydrogenase mRNA. Mol Cell Endocrinol. 1995;109:183-8.

163. Tchernof A, Belanger C, Morisset AS, et al. Regional differences in adipose tissue metabolism in women: minor effect of obesity and body fat distribution. Diabetes. 2006;55:1353-60.

164. Jensen MD, Cardin S, Edgerton D, et al. Splanchnic free fatty acid kinetics. Am J Physiol Endocrinol Metab. 2003;284:E1140-8.

165. Hocking SL, Wu LE, Guilhaus M, et al. Intrinsic depot-specific differences in the secretome of adipose tissue, preadipocytes, and adipose tissue-derived microvascular endothelial cells. Diabetes. 2010;59:3008-16.

166. Nielsen S, Guo Z, Johnson CM, et al. Splanchnic lipolysis in human obesity. J Clin Invest. 2004;113:1582-8.

167. Cheung O, Kapoor A, Puri P, et al. The impact of fat distribution on the severity of nonalcoholic fatty liver disease and metabolic syndrome. Hepatology. 2007;46:1091-100.

168. Garaulet M, Perez-Llamas F, Baraza JC, et al. Body fat distribution in pre-and post-menopausal women: metabolic and anthropometric variables. J Nutr Health Aging. 2002;6:123-6.

169. Kvist H, Chowdhury B, Grangard U, et al. Total and visceral adipose-tissue volumes derived from measurements with computed tomography in adult men and women: predictive equations. Am J Clin Nutr. 1988;48:1351-61. 
170. Italian Association for the Study of the Liver, Lonardo A, Nascimbeni F, et al. AISF position paper on nonalcoholic fatty liver disease (NAFLD): updates and future directions. Dig Liver Dis. 2017;49:471-483.

171. Paquette A, Wang D, Jankowski M, et al. Effects of ovariectomy on PPAR alpha, SREBP-1c, and SCD-1 gene expression in the rat liver. Menopause. 2008;15:1169-75.

172. Spangenburg EE, Wohlers LM, Valencia AP. Metabolic dysfunction under reduced estrogen levels: looking to exercise for prevention. Exerc Sport Sci Rev. 2012;40:195-203.

173. Domingos MM, Rodrigues MF, Stotzer US, et al. Resistance training restores the gene expression of molecules related to fat oxidation and lipogenesis in the liver of ovariectomized rats. Eur J Appl Physiol. 2012;112:1437-44.

174. Lundholm L, Zang H, Hirschberg AL, et al. Key lipogenic gene expression can be decreased by estrogen in human adipose tissue. Fertil Steril. 2008;90:44-8.

175. Pighon A, Gutkowska J, Jankowski M, et al. Exercise training in ovariectomized rats stimulates estrogenic-like effects on expression of genes involved in lipid accumulation and subclinical inflammation in liver. Metabolism. 2011;60:629-39.

176. Li ZZ, Berk M, McIntyre TM, et al. Hepatic lipid partitioning and liver damage in nonalcoholic fatty liver disease: role of stearoyl-CoA desaturase. J Biol Chem. 2009;284:5637-44.

177. Liu X, Burhans MS, Flowers MT, et al. Hepatic oleate regulates liver stress response partially through PGC-1alpha during high-carbohydrate feeding. J Hepatol. 2016;65:103-12.

178. Marks KA, Kitson AP, Shaw B, et al. Stearoyl-CoA desaturase 1, elongase 6 and their fatty acid products and precursors are altered in ovariectomized rats with 17 beta-estradiol and progesterone treatment. Prostaglandins Leukot Essent Fatty Acids. 2013;89:89-96.

179. Park HG, Kothapalli KS, Park WJ, et al. Palmitic acid (16:0) competes with omega-6 linoleic and omega-3 a-linolenic acids for FADS2 mediated delta6-desaturation. Biochim Biophys Acta. 2016;1861:91-7.

180. Jump DB, Depner CM, Tripathy S, et al. Potential for dietary omega-3 fatty acids to prevent nonalcoholic fatty liver disease and reduce the risk of primary liver cancer. Adv Nutr. 2015;6:694-702.

181. Childs CE, Romeu-Nadal M, Burdge GC, et al. Gender differences in the n-3 fatty acid content of tissues. Proc Nutr Soc. 2008;67:19-27.
182. Yoon M. PPARalpha in obesity: sex difference and estrogen involvement. PPAR Res. 2010. doi:10. $1155 / 2010 / 584296$.

183. Romano KA, Vivas EI, Amador-Noguez D, et al. Intestinal microbiota composition modulates choline bioavailability from diet and accumulation of the proatherogenic metabolite trimethylamine-N-oxide. MBio. 2015;6:e02481.

184. McCarty MF, DiNicolantonio JJ. An increased need for dietary cysteine in support of glutathione synthesis may underlie the increased risk for mortality associated with low protein intake in the elderly. Age (Dordr). 2015;37:96.

185. Fischer LM, daCosta KA, Kwock L, et al. Sex and menopausal status influence human dietary requirements for the nutrient choline. Am J Clin Nutr. 2007;85:1275-85.

186. Inaba K, Suzuki S, Ihara H, et al. Sexual dimorphism in endotoxin susceptibility after partial hepatectomy in rats. J Hepatol. 2005;42:719-27.

187. Hotamisligil GS. Inflammation, metaflammation and immunometabolic disorders. Nature. 2017;542:177-85.

188. Voysey M, Barker CI, Snape MD, et al. Sex-dependent immune responses to infant vaccination: an individual participant data meta-analysis of antibody and memory B cells. Vaccine. 2016;34:1657-64.

189. Syn WK, Jung Y, Omenetti A, et al. Hedgehog-mediated epithelial-to-mesenchymal transition and fibrogenic repair in nonalcoholic fatty liver disease. Gastroenterology. 2009;137(1478-1488):e8.

190. Myers BR, Sever N, Chong YC, et al. Hedgehog pathway modulation by multiple lipid binding sites on the smoothened effector of signal response. Dev Cell. 2013;26:346-57.

191. Pospisilik JA, Schramek D, Schnidar H, et al. Drosophila genome-wide obesity screen reveals hedgehog as a determinant of brown versus white adipose cell fate. Cell. 2010;140:148-60.

192. Teperino R, Amann S, Bayer M, et al. Hedgehog partial agonism drives Warburg-like metabolism in muscle and brown fat. Cell. 2012;151:414-26.

193. Chung SI, Moon H, Ju HL, et al. Hepatic expression of Sonic Hedgehog induces liver fibrosis and promotes hepatocarcinogenesis in a transgenic mouse model. J Hepatol. 2016;64:618-27.

194. Wehner D, Weidinger G. Signaling networks organizing regenerative growth of the zebrafish fin. Trends Genet. 2015;31:336-43. 
195. Jerde TJ. Phosphatase and tensin homologue: novel regulation by developmental signaling. J Signal Transduct. 2015;2015:282567.

196. Borggrefe T, Lauth M, Zwijsen A, et al. The Notch intracellular domain integrates signals from Wnt, Hedgehog, TGFbeta/BMP and hypoxia pathways. Biochim Biophys Acta. 2016;1863:303-13.

197. Shi Y, Chen J, Karner CM, et al. Hedgehog signaling activates a positive feedback mechanism involving insulin-like growth factors to induce osteoblast differentiation. Proc Natl Acad Sci USA. 2015;112:4678-83.

198. Varelas X. The Hippo pathway effectors TAZ and YAP in development, homeostasis and disease. Development. 2014;141:1614-26.

199. Moeini A, Cornella H, Villanueva A. Emerging signaling pathways in hepatocellular carcinoma. Liver Cancer. 2012;1:83-93.

200. Guy CD, Suzuki A, Zdanowicz M, et al. Hedgehog pathway activation parallels histologic severity of injury and fibrosis in human nonalcoholic fatty liver disease. Hepatology. 2012;55:1711-21.

201. Varlamov O, Bethea CL, Roberts CT Jr. Sex-specific differences in lipid and glucose metabolism. Front Endocrinol (Lausanne). 2014;5:241.

202. Link JC, Chen X, Arnold AP, et al. Metabolic impact of sex chromosomes. Adipocyte. 2013;2:74-9.

203. Suzuki A, Abdelmalek MF. Nonalcoholic fatty liver disease in women. Womens Health (Lond). 2009;5:191-203.

204. Yasuda M, Shimizu I, Shiba M, et al. Suppressive effects of estradiol on dimethylnitrosamine-induced fibrosis of the liver in rats. Hepatology. 1999;29:719-27.

205. Besse-Patin A, Leveille M, Oropeza D, et al. Estrogen signals through peroxisome proliferator-activated receptor-gamma coactivator 1alpha to reduce oxidative damage associated with diet-induced fatty liver disease. Gastroenterology 2017;152:243-256.

206. Zhang Y, Wu L, Wang Y, et al. Protective role of estrogen-induced miRNA-29 expression in carbon tetrachloride-induced mouse liver injury. J Biol Chem. 2012;287:14851-62.

207. Zhang ZC, Liu Y, Xiao LL, et al. Upregulation of miR-125b by estrogen protects against non-alcoholic fatty liver in female mice. J Hepatol. 2015;63:1466-75.

208. Ricchi M, Bertolotti M, Anzivino C, et al. 17 Beta-estradiol prevents cytotoxicity from hydrophobic bile acids in HepG2 and WRL-68 cell cultures. J Gastroenterol Hepatol. 2006;21:894-901.

209. Chambliss KL, Barrera J, Umetani M, et al. Nonnuclear estrogen receptor activation improves hepatic steatosis in female mice. Endocrinology. 2016;157:3731-41.

210. Nemoto Y, Toda K, Ono M, et al. Altered expression of fatty acid-metabolizing enzymes in aromatase-deficient mice. J Clin Invest. 2000;105:1819-25.

211. Heine PA, Taylor JA, Iwamoto GA, et al. Increased adipose tissue in male and female estrogen receptor-alpha knockout mice. Proc Natl Acad Sci USA. 2000;97:12729-34.

212. Lemieux C, Phaneuf D, Labrie F, et al. Estrogen receptor alpha-mediated adiposity-lowering and hypocholesterolemic actions of the selective estrogen receptor modulator acolbifene. Int $\mathrm{J}$ Obes (Lond). 2005;29:1236-44.

213. Saphner T, Triest-Robertson S, Li H, et al. The association of nonalcoholic steatohepatitis and tamoxifen in patients with breast cancer. Cancer. 2009;115:3189-95.

214. Luo F, Ishigami M, Achiwa $\mathrm{K}$, et al. Raloxifene ameliorates liver fibrosis of nonalcoholic steatohepatitis induced by choline-deficient high-fat diet in ovariectomized mice. Dig Dis Sci. 2015;60:2730-9.

215. Clark JM, Brancati FL, Diehl AM. Nonalcoholic fatty liver disease. Gastroenterology. 2002;122:1649-57.

216. Vetelainen R, van Vliet A, van Gulik TM. Essential pathogenic and metabolic differences in steatosis induced by choline or methione-choline deficient diets in a rat model. J Gastroenterol Hepatol. 2007;22:1526-33.

217. Yu D, Shu XO, Xiang YB, et al. Higher dietary choline intake is associated with lower risk of nonalcoholic fatty liver in normal-weight Chinese women. J Nutr. 2014;144:2034-40.

218. Guerrerio AL, Colvin RM, Schwartz AK, et al. Choline intake in a large cohort of patients with nonalcoholic fatty liver disease. Am J Clin Nutr. 2012;95:892-900.

219. Buchman AL, Dubin MD, Moukarzel AA, et al. Choline deficiency: a cause of hepatic steatosis during parenteral nutrition that can be reversed with intravenous choline supplementation. Hepatology. 1995;22:1399-403.

220. Vilar-Gomez E, Martinez-Perez Y, Calzadilla-Bertot $\mathrm{L}$, et al. Weight loss through lifestyle modification 
significantly reduces features of nonalcoholic steatohepatitis. Gastroenterology. 2015;149(367-78):e5 (quiz e14-5).

221. Orci LA, Gariani K, Oldani G, et al. Exercise-based interventions for nonalcoholic fatty liver disease: a meta-analysis and meta-regression. Clin Gastroenterol Hepatol. 2016;14:1398-411.

222. Hashida R, Kawaguchi T, Bekki M, et al. Aerobic vs. resistance exercise in non-alcoholic fatty liver disease: A systematic review. J Hepatol. 2017;66:142-152.

223. Astrup A. Physical activity and weight gain and fat distribution changes with menopause: current evidence and research issues. Med Sci Sports Exerc. 1999;31:S564-7.

224. Barsalani R, Riesco E, Lavoie JM, et al. Effect of exercise training and isoflavones on hepatic steatosis in overweight postmenopausal women. Climacteric. 2013;16:88-95.
225. Rezende RE, Duarte SM, Stefano JT, et al. Randomized clinical trial: benefits of aerobic physical activity for 24 weeks in postmenopausal women with nonalcoholic fatty liver disease. Menopause. 2016;23:876-83.

226. Rossouw JE, Anderson GL, Prentice RL, et al. Risks and benefits of estrogen plus progestin in healthy postmenopausal women: principal results from the Women's Health Initiative randomized controlled trial. JAMA. 2002;288:321-33.

227. Beral V, Million Women Study Collaborators. Breast cancer and hormone-replacement therapy in the Million Women Study. Lancet. 2003;362:419-27.

228. Loria P, Carulli L, Bertolotti M, et al. Endocrine and liver interaction: the role of endocrine pathways in NASH. Nat Rev Gastroenterol Hepatol. 2009;6:236-47. 